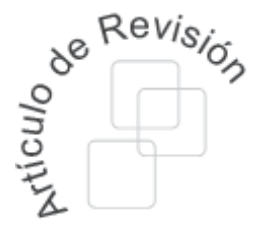

\title{
Una revisión al estado del arte de la integración de toma de decisiones en la red logística
}

\section{State of the art review of the integration of decision making in the logistics network}

\section{Resumen}

El presente artículo es resultado de la investigación del proyecto titulado "Integración de Toma de decisiones en la red logística" y comprende la revisión del estado del arte en el cual se identifica el concepto de integración en la cadena de suministro, los tipos de integración, mecanismos de integración, herramientas de integración, estrategias de integración y finalmente los beneficios y las medidas de rendimiento.

Palabras clave: Integración de la cadena de suministro, tecnologías de información, arcos de integración, outsourcing de servicios.

\section{Abstract}

This article is the result of the research project entitled "Integration of Decision Making in the logistics network". It conduct a review of the state of art in which the concept of integration in the supply chain is identified, as well as the types of integration, mechanisms of integration, integration tools, integration strategies and finally the benefits and performance measures.

Key words: Integration of the supply chain, information technologies, arcs of integration, outsourcing services.

\section{Introducción}

En el escenario de la manufactura global, las decisiones en la cadena de suministro no se pueden tomar por separado. Hoy en día estas decisiones tienen que ver con los procesos de integración dentro y fuera de la empresa. Proveedores, plantas de manufactura, centros de servicio, 
operadores logísticos, contratistas, distribuidores, minoristas, detallistas, en fin, todos los actores de una red logística o de redes logísticas tienen aportan a los procesos de integración y asimismo a tomar decisiones conjuntas que beneficien a la red y a todos los actores que la integran (Taskin Gumus, 2007), (Ahmed Musa, 2013).

Los procesos de integración de decisiones en la red logística se apoyan en gran medida con el avance de las tecnologías de la información, esto hace que la manufactura global esté dentro del alcance de muchas organizaciones pequeñas que entrarán a engrosar la alta competencia de mercado (Vernadat, 2002), (Brent D. Williams, 2013).

Una de las decisiones conjuntas de gran trascendencia es la minimización de los costos logísticos. Los costos logísticos dominan los gastos de las empresas manufactureras. Por lo tanto, las decisiones logísticas involucran la obtención de la materia prima, producción y distribución y estas decisiones no se deben hacer por separado sino integradas con el fin de obtener ahorros. Para maximizar los ahorros, varias investigaciones han propuesto la integración de las decisiones logísticas de una red producción-distribución dentro de un ambiente de manufactura global. Así, una empresa de manufactura global puede ser formulado como una combinación red producción-distribución constituida de múltiples fuentes y múltiples destinos (Pagell, 2004), (Christina W.Y. Wong, 2013).

En recientes estudios, los modelos para coordinar redes de sistemas de produccióndistribución han tendido a focalizar la integración de decisiones en programación de transporte, políticas y control de inventarios y programación de tamaños de lotes de producción (Ahmed Musa, 2013), (Brent D. Williams, 2013).

El presente artículo se enfoca específicamente en describir el estado del arte en cuanto a la integración de la toma decisiones en la cadena de suministro desde sus conceptos primarios hasta la justificación de la integración.

\section{Metodología}

Para abordar el estado del arte en cuanto a la Integración de la Toma de decisiones en la Red Logística se consultaron diferentes bases de datos bibliográficas (Scopus, ScienceDirect, SpringerLink, Proquest, IEEE, IOPSCIENCE). De los artículos encontrados, se seleccionaron los artículos que tratan específicamente el tema. Para construir el estado del arte de la integración de toma de decisiones en la red logística, se requirió responder las siguientes preguntas: ¿Qué se entiende por integración?, ¿Qué se puede integrar?, ¿Qué tipos de integración existen? ¿Cómo se integra?, ¿Qué se necesita integrar? , ¿Para qué se integra? ¿¿Qué estrategias de integración existen? ¿Cómo se mide la integración? Con base en estas preguntas se estructuró el estado del arte como se expone en la Sección 3. 


\section{Marco teórico}

La integración de la cadena de suministro ha sido tratada desde diferentes perspectivas. Es así, como se distinguen la integración con clientes, integración de la información, integración logística y de distribución e integración con proveedores (Vijayasarathy, 2010), (Christina W.Y. Wong, 2013), (Claudia Colicchia, 2013), (Danese, 2013).

\section{1. ¿Qué se entiende por integración?}

Para definir qué es integración, no existe una definición única y aceptada o puesta en funcionamiento. La literatura e investigación sobre el concepto de integración de la cadena de suministro es desarrollada por varios investigadores. Entre las definiciones más conocidas podemos citar las siguientes (Pagell, 2004), (Xiande Zhao, 2008), (Devendra Choudhary, 2013), (Edrisi Muñoz, 2013):

- Kahn y Mentzer (1998) y Pinedo (2005) definen la integración como el proceso de integración entre los departamentos y la colaboración interdepartamental que aporta a una organización cohesiva.

- O’Leary Kelly y Flores (2002) afirman que la integración se refiere al grado en el cual las partes trabajan juntas de manera cooperativa para llegar a resultados mutuamente aceptables.

- O’Leary Kelly y Flores (2002) y Kahn y Mentzer (1998) a partir de un esfuerzo de investigación definieron la integración así: "la integración es un proceso de interacción y colaboración en el que la fabricación, compras y logística trabajan juntos de manera cooperativa para llegar a resultados mutuamente aceptables para su organización".

- Según Bowersox y Muran (1989), Hillerbrad y Braman (2003), definen la integración de la cadena de suministro como el grado en que el fabricante colabora en forma estratégica con sus socios de la cadena de suministro y gestiona los procesos de colaboración intra e inter-organizacional.

- Stank et.al (2001), enuncia que la integración de la cadena de suministro consiste en la integración interna de diferentes funciones dentro de una compañía e integración externa con los socios comerciales.

Si bien el concepto básico de la integración puede ser familiar para la mayoría de los investigadores, las operacionalizaciones reales de la construcción del concepto de integración serian muchas. La siguiente Tabla I, resume los estudios representativos relacionados con la integración y las operacionalizaciones de la construcción utilizadas en estos estudios (J.F., 1981), (Chien, 1993), (Speranza, 1994), (Hall, 1996), (Thomas, 1996), (Fumero, 1997), (Bookbinder, 1999), (Pagell, 2004), (Sanchez, 2006), (Farahani, 2013). 


\begin{tabular}{|c|c|c|c|}
\hline Referencia & Enfoque del estudio & $\begin{array}{l}\text { Nivel de } \\
\text { análisis }\end{array}$ & Definición \\
\hline $\begin{array}{l}\text { Ettlie y Stoll } \\
(1990)\end{array}$ & $\begin{array}{l}\text { Desarrollo de } \\
\text { productos }\end{array}$ & Interno & Coordinación y colaboración \\
\hline Alder (1992) & $\begin{array}{l}\text { Desarrollo de } \\
\text { productos }\end{array}$ & Interno & Coordinación \\
\hline $\begin{array}{l}\text { Sussman y } \\
\text { Dean (1992) }\end{array}$ & $\begin{array}{l}\text { Desarrollo de } \\
\text { productos }\end{array}$ & Interno & Dos funciones de trabajo en conjunto \\
\hline $\begin{array}{l}\text { Wheelwright y } \\
\text { Clark (2005) }\end{array}$ & $\begin{array}{l}\text { Desarrollo de } \\
\text { productos }\end{array}$ & Interno & $\begin{array}{l}\text { Integración entre los ingenieros de diseño con los } \\
\text { ingenieros de procesos para resolver problemas en el } \\
\text { desarrollo de productos }\end{array}$ \\
\hline $\begin{array}{l}\text { Kahn y McDo- } \\
\text { nough (1997) }\end{array}$ & $\begin{array}{l}\text { Manufactura y } \\
\text { mercadeo }\end{array}$ & Interno & Dos componentes que se integran e interactúan \\
\hline $\begin{array}{l}\text { Mintzberg et.al. } \\
\text { (1996) }\end{array}$ & $\begin{array}{l}\text { Todas las formas de } \\
\text { colaboración }\end{array}$ & $\begin{array}{l}\text { Interno y } \\
\text { externo }\end{array}$ & Colaboración = Integración \\
\hline $\begin{array}{l}\text { Kahn y Mentzer } \\
\text { (1998) }\end{array}$ & $\begin{array}{l}\text { Mercadeo y otros } \\
\text { departamentos }\end{array}$ & Interno & $\begin{array}{l}\text { Interacción y colaboración (Kahn y McDonough, 1997). } \\
\text { La integración se define formalmente como un proceso } \\
\text { de interacción y colaboración interdepartamental. }\end{array}$ \\
\hline $\begin{array}{l}\text { Ellinger et.al. } \\
(2000)\end{array}$ & Mercadeo y logística & Interno & $\begin{array}{l}\text { Kahn y McDonough (1997) -dos grandes dimensiones- } \\
\text { colaboración e interacción }\end{array}$ \\
\hline $\begin{array}{l}\text { Frolich y Wes- } \\
\text { brook (2001) }\end{array}$ & $\begin{array}{l}\text { Cadena de sumi- } \\
\text { nistro }\end{array}$ & Externo & Coordinación \\
\hline $\begin{array}{l}\text { Krajewskis y } \\
\text { Wei (2001) }\end{array}$ & $\begin{array}{l}\text { Proveedor com- } \\
\text { prador }\end{array}$ & Externo & Colaboración y coordinación \\
\hline $\begin{array}{l}\text { Narasinham y } \\
\text { Das (2001) }\end{array}$ & $\begin{array}{l}\text { Compras en la } \\
\text { empresa }\end{array}$ & Interno & $\begin{array}{l}\text { La integración de compras implica la participación } \\
\text { activa de la empresa y tiene como objetivo promover la } \\
\text { alineación de las metas y prácticas de compras con las } \\
\text { prioridades estratégicas de negocio }\end{array}$ \\
\hline $\begin{array}{l}\text { Verma et.al. } \\
\text { (2001) }\end{array}$ & $\begin{array}{l}\text { Operaciones y } \\
\text { mercadeo }\end{array}$ & Interno & $\begin{array}{l}\text { El enfoque de trabajar en forma conjunta las operacio- } \\
\text { nes y el marketing mejoraran sus propias funciones y } \\
\text { relaciones. }\end{array}$ \\
\hline $\begin{array}{l}\text { Ganeshan } \\
(2002)\end{array}$ & $\begin{array}{l}\text { Papel de la } \\
\text { tecnología Internet } \\
\text { en la cadena de } \\
\text { suministro }\end{array}$ & $\begin{array}{l}\text { Cadena de } \\
\text { suministro } \\
\text { (interno y } \\
\text { externo) }\end{array}$ & Plataforma tecnológica compartida \\
\hline $\begin{array}{l}\text { Gerwin y } \\
\text { Barrowman } \\
(2002)\end{array}$ & $\begin{array}{l}\text { Desarrollo de } \\
\text { productos }\end{array}$ & Interno & $\begin{array}{l}\text { IPD es un enfoque de gestión para mejorar el rendimien- } \\
\text { to del desarrollo de nuevos productos }\end{array}$ \\
\hline Kelly (2002) & ERP & $\begin{array}{l}\text { Cadena de } \\
\text { suministro } \\
\text { (Interno y } \\
\text { externo) }\end{array}$ & Plataforma tecnológica compartida \\
\hline
\end{tabular}




\begin{tabular}{|l|l|l|l|}
\hline $\begin{array}{l}\text { Naransinham y } \\
\text { Kim (2001) }\end{array}$ & $\begin{array}{l}\text { Cadena de sumi- } \\
\text { nistro }\end{array}$ & $\begin{array}{l}\text { Interno y } \\
\text { externo }\end{array}$ & Coordinación \\
\hline $\begin{array}{l}\text { O'Leary-Kelly y } \\
\text { Flores (2002) }\end{array}$ & $\begin{array}{l}\text { Manufactura/Mer- } \\
\text { cados }\end{array}$ & Interno & $\begin{array}{l}\text { La integración se refiere a la medida en que las partes } \\
\text { separadas trabajan juntos en forma cooperativa para } \\
\text { legar a resultados aceptables para ambas partes. En } \\
\text { consecuencia, esta definición abarca construcciones } \\
\text { relacionadas con el grado de cooperación, coordinación, } \\
\text { interacción y colaboración }\end{array}$ \\
\hline Swan (2002) & $\begin{array}{l}\text { Desarrollo de } \\
\text { productos }\end{array}$ & Interno & Coordinación \\
\hline
\end{tabular}

Existe un amplio cuerpo de investigación sobre las relaciones unidimensionales de la Integración de la cadena de suministro, examinando las relaciones de colaboración entre el fabricante y/o clientes o proveedores (Paulra, et.al 2008; Mabert y Ventietaramanan, 1998; Spekman et.al. 1998; Fawcett y Magnan, 2002). Mientras que algunos se centran en las relaciones diádicas con socios de la cadena de suministro (Lee y Whang, 2001), otros se centran en la gestión de la cadena de suministro como un sistema único, en lugar de intentar optimizar individualmente los subsistemas fragmentados (Vickery et.al. 2003; Naylor et.al. 1999; Bowersox y Murach, 1989; Hammer, 1990; Stevens, 1989). Si bien algunas definiciones de la integración de la cadena de suministro (SCI) hacen énfasis en los flujos de materiales y piezas, otros se centran en los flujos de información, recursos y dinero en efectivo. Se construye sobre la literatura existente de la integración de la cadena de suministro, incluido el fabricante (integración interna) y se extiende en ambas direcciones (integración de clientes y proveedores) (Blumenfield, 1985), (Benjamin, 1989), (Sharp, 1989), (Cohen, 1988), (Blumenfield, 1991), (Neal Juster, 1997), (Wang Sena, 2004), (Pagell, 2004), (Ferdinand Jaspers, 2006), (Farahani, 2013).

\section{2. ¿Qué se puede integrar?}

Para responder a la pregunta ¿Qué se puede integrar?, evidentemente se relaciona con la pregunta ¿Qué se puede coordinar en la cadena de suministro o red logística? Los artículos que abordan la planeación coordinada entre dos o más etapas de la SC (Supply Chain) se clasifican según la Figura 1 (Arshinder, 2008).

De acuerdo al tipo de coordinación se relacionan los ítems (inventarios, producción, lotes de producción, pedidos, transporte, distribución, almacenamiento, etc) que se pueden integrar. Algunos de estos tipos de coordinación se describen a continuación (Mithun Sharma, 2008), (Arshinder, 2008):

- Coordinación entre Producción y Distribución. La integración de estas dos funciones puede dar lugar a un ahorro sustancial en los costos globales y una mejora en el servicio mediante la explotación de economías de escala de producción y transporte. Algunos de los ítems que se pueden integrar son: El lote de producción, Cargas de vehículos, Control y manejo de los inventarios, Rutas de 


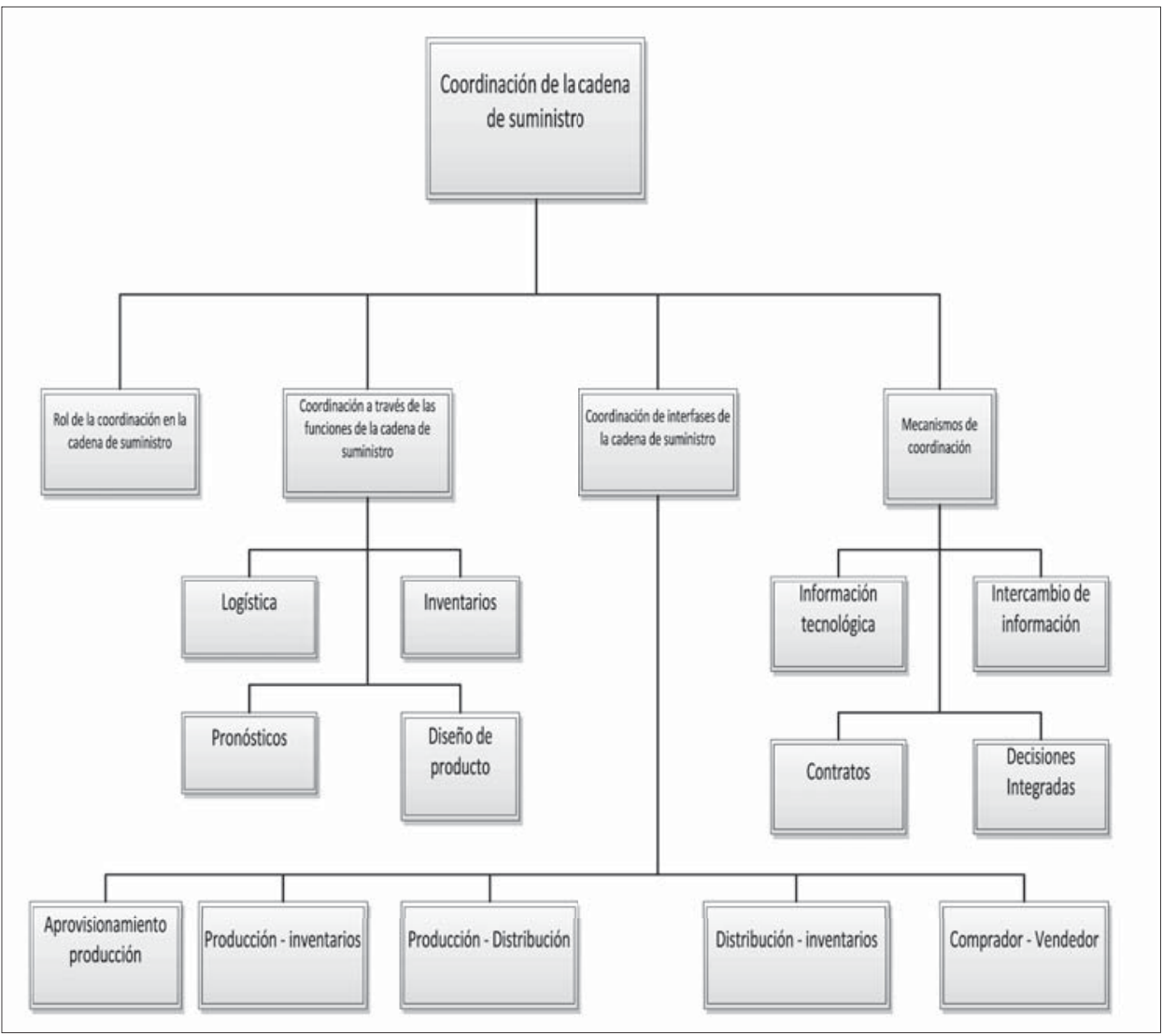

Figura 1. Taxonomía Tipos de Coordinación de la cadena de suministro. Fuente: (Arshinder, 2008)

distribución, Longitud del ciclo de producción, Frecuencia de pedidos, Tamaño del pedido, Asignación de producción.

- Coordinación entre aprovisionamiento y producción. Goyal y Deshmukh (1992) revisaron la literatura sobre los sistemas integrados de producción y aprovisionamiento (IPP). Los diferentes modelos IPP se clasificaron en categorías basadas en el número de productos, horizonte de planeación, métodos de solución empleados, ordenes de reposición y soluciones algorítmicas. Algunos de los ítems que se pueden integrar son: Control y manejo de inventarios, Frecuencia de pedidos de materia de prima o partes de ensamble, Tamaño de los pedidos de la materia prima o partes de ensamble.

- Coordinación entre producción e inventario. Lu (1995) consideró el enfoque heurístico del problema para un único vendedor con múltiples compradores con envíos de igual tamaño. Con la coordinación de las reposiciones de los distintos elementos, el vendedor puede reducir su costo total anual en un $30 \%$. Los compradores también se benefician del modelo multi-comprador, redu- 
ciendo sus costos. Algunos de los ítems que se pueden integrar son: Reposiciones, Políticas de inventario, Niveles de reorden, Stock de seguridad, Tiempos de suministro.

- Coordinación entre distribución e inventario. Jayaraman (1998) desarrollaron un modelo integrado de programación matemática mixta-entera para la minimización del costo total de distribución asociado con tres componentes de decisión: ubicación de las instalaciones, los parámetros de inventarios y alternativas de selección de transporte. Yokoyama (2002) desarrolló un modelo de optimización integrada del sistema producción-inventario en la que cualquier punto de consumo puede ser suministrado por los centros de distribución multiples. Las políticas de inventario de revisión periódica y el problema de transporte se consideran al mismo tiempo. Algunos de los ítems que se pueden integrar son: Ubicación de instalaciones (centros de acopio, almacenes, bodegas, centros de distribución), Parámetros de inventarios, Modos de transporte, Selección de rutas, Cantidades a transportar, Número de envíos, Cantidad a ordenar.

Diversas perspectivas se presentan en la literatura sobre la coordinación de la cadena de suministro. La Tabla II resume varias perspectivas de la coordinación de la cadena suministro (Arshinder, 2008).

Los problemas de coordinación en las interfaces de la cadena de suministro se presentan en la Tabla III. Sharufali (2000) hizo énfasis en las ventajas de los mecanismos de coordinación tales como cambios en los precios, descuentos por cantidad (Sarmath et.al 2007), entregas parciales y el establecimiento de políticas conjuntas en el contexto de las empresas manufactureras. Sahin y Robinson (2002) revisaron la literatura a nivel operativo para estudiar el impacto de la información en tiempo real y la coordinación en la toma de decisiones con información distorsionada, variabilidad de la demanda, falta de precisión en los pronósticos, políticas de inventario y factores de inversión en un ambiente multi-eslabón y multi-periodo (Arshinder, 2008).

En forma general, según Vernadat (2002), las necesidades de integración en la cadena de suministro surgen de los problemas de integración desde diferentes perspectivas (Vernadat, 2002), (Erhan Kutanoglu, 2008):

- Integración de los mercados: Nuevas zonas comerciales y economías libres se están estableciendo en todo el mundo (Unión Europea, Mercosur, Mercado Indonesio).

- Integración entre varios sitios de fabricación y la investigación y desarrollo: Se refiere a proyectos de colaboración entre empresas que contribuyen al desarrollo de productos complejos.

- Integración entre proveedores y fabricantes: Para reducir los plazos de entrega de productos y compartir los riesgos en el lanzamiento de nuevos productos, los fabricantes y proveedores deben integrar y sincronizar sus procesos. 
Tabla II. Taxonomía - Perspectivas de la coordinación de la cadena de suministro

\begin{tabular}{|c|c|c|}
\hline AUTOR (AÑO) & PERSPECTIVA & CONTEXTO \\
\hline $\begin{array}{l}\text { Narus y } \\
\text { Anderson } \\
(1996)\end{array}$ & $\begin{array}{l}\text { La cooperación entre empresas independientes, pero relacionadas para } \\
\text { compartir recursos y capacidades y satisfacer las necesidades más extraor- } \\
\text { dinarias de sus clientes }\end{array}$ & $\begin{array}{l}\text { Distribución de los } \\
\text { recursos }\end{array}$ \\
\hline $\begin{array}{c}\text { Lambert } \\
\text { et.al. (1999) }\end{array}$ & $\begin{array}{l}\text { Un grado particular de relación entre los miembros de la cadena como un } \\
\text { medio para compartir los riesgos y beneficios que se derivan de los resul- } \\
\text { tados empresariales. }\end{array}$ & $\begin{array}{l}\text { Compartir riesgos y } \\
\text { recompensas }\end{array}$ \\
\hline $\begin{array}{l}\text { Ballou et.al. } \\
\text { (2000) }\end{array}$ & $\begin{array}{l}\text { La capacidad de la función logística para integrar las actividades de la ca- } \\
\text { dena de suministro entre sí a través de diferentes líneas de autoridad y res- } \\
\text { ponsabilidad organizativa }\end{array}$ & Responsabilidad \\
\hline $\begin{array}{l}\text { Larsen } \\
(2000)\end{array}$ & $\begin{array}{l}\text { El trabajo colaborativo para la planificación conjunta, el desarrollo conjunto } \\
\text { de productos, el intercambio de información mutua y sistema de información } \\
\text { integrados, la coordinación entre diversos niveles en las empresas de la red, } \\
\text { la cooperación a largo plazo y el reparto equitativo de los riesgos y beneficios }\end{array}$ & $\begin{array}{l}\text { Visión holística de la } \\
\text { coordinación }\end{array}$ \\
\hline Lee (2000) & $\begin{array}{l}\text { Coordinación de la cadena de suministro como vehículo para el rediseño } \\
\text { de la toma de decisiones, flujo de trabajo y los recursos entre los miembros } \\
\text { de la cadena con el objeto de mejorar el rendimiento de la cadena de su- } \\
\text { ministro }\end{array}$ & $\begin{array}{l}\text { Flujo de trabajo y } \\
\text { dependencia de los } \\
\text { recursos }\end{array}$ \\
\hline $\begin{array}{l}\text { Simatupang } \\
\text { et.al. (2002) }\end{array}$ & $\begin{array}{l}\text { Dada la naturaleza de las interdependencias entre las unidades que confor- } \\
\text { man la cadena de suministro, la coordinación es un requisito previo necesa- } \\
\text { rio para integrar sus operaciones, para lograr el objetivo común de la cadena } \\
\text { de suministro en su conjunto }\end{array}$ & Mutualidad \\
\hline $\begin{array}{l}\text { Larsen et.al. } \\
\text { (2003) }\end{array}$ & $\begin{array}{l}\text { Cuando dos o más partes en la cadena de suministro planean conjuntamen- } \\
\text { te una serie de actividades de promoción y elaboran proyecciones sincroni- } \\
\text { zadas, sobre la base de los cuales se determinan los procesos de producción } \\
\text { y reabastecimiento }\end{array}$ & $\begin{array}{l}\text { Actividades de pro- } \\
\text { moción conjuntas, } \\
\text { pronósticos }\end{array}$ \\
\hline $\begin{array}{l}\text { Hill y Omar } \\
\text { (2006) }\end{array}$ & $\begin{array}{l}\text { La coordinación puede lograrse cuando los miembros de la cadena de su- } \\
\text { ministro minimizan conjuntamente los costos de operación y comparten } \\
\text { los beneficios después de planear conjuntamente la producción y definir } \\
\text { sus políticas }\end{array}$ & $\begin{array}{l}\text { Toma de decisiones } \\
\text { conjunta y partici- } \\
\text { pación en los bene- } \\
\text { ficios }\end{array}$ \\
\hline
\end{tabular}

Fuente: (Arshinder, 2008)

- Integración del diseño y la fabricación: Para reducir el tiempo de lanzamiento al mercado y reducir al mínimo los errores de diseño. Las prácticas de ingeniería concurrente se deben implementar para obtener una mayor integración entre las actividades de diseño y fabricación.

- Integración de múltiples proveedores de hardware y software: las arquitecturas de sistemas abiertos son necesarios para proporcionar la interoperabilidad de las diferentes soluciones informáticas de hardware y software de uso común en entornos industriales 
Tabla III . Taxonomía - Coordinación de varias actividades e interfaces en la cadena de suministro. Fuente: (Arshinder, 2008)

\begin{tabular}{|c|c|c|c|c|c|}
\hline \multicolumn{6}{|c|}{ Coordinacion de varias actividades e interfaces de la cadena de suministro } \\
\hline Autor & $\begin{array}{l}\text { Problema de } \\
\text { coordinacion }\end{array}$ & $\begin{array}{c}\text { Mecanismo } \\
\text { de coordinacion }\end{array}$ & $\begin{array}{l}\text { Estructura } \\
\text { cadena } \\
\text { suministro }\end{array}$ & Metodologia usada & $\begin{array}{c}\text { Medidas } \\
\text { de } \\
\text { rendimiento }\end{array}$ \\
\hline \multicolumn{6}{|c|}{ LOGISTICA } \\
\hline $\begin{array}{l}\text { Stank } \\
\text { et.al } \\
(1999)\end{array}$ & $\begin{array}{l}\text { Diferentes objetivos } \\
\text { entre el transportis- } \\
\text { ta y el proveedor de } \\
\text { transporte }\end{array}$ & $\begin{array}{l}\text { Intercambio de } \\
\text { información y tec- } \\
\text { nologías de infor- } \\
\text { mación }\end{array}$ & $\begin{array}{l}\text { Logística Pro- } \\
\text { veedores y } \\
\text { clientes }\end{array}$ & $\begin{array}{l}\text { Estudio } \\
\text { empírico }\end{array}$ & $\begin{array}{l}\text { Nivel de inventarios, } \\
\text { costos (transporte, } \\
\text { almacenamiento, } \\
\text { ordenar), variación } \\
\text { ciclos de los pedidos, } \\
\text { entregas a tiempo }\end{array}$ \\
\hline $\begin{array}{l}\text { Stank y } \\
\text { Goldsby } \\
(2000)\end{array}$ & $\begin{array}{l}\text { Diferentes objetivos } \\
\text { entre el transportis- } \\
\text { ta y el proveedor de } \\
\text { transporte }\end{array}$ & $\begin{array}{l}\text { Intercambio de } \\
\text { información , ali- } \\
\text { neación de metas, } \\
\text { EDI, Contratos }\end{array}$ & $\begin{array}{l}\text { Logística Pro- } \\
\text { veedores y } \\
\text { clientes }\end{array}$ & $\begin{array}{c}\text { Marco } \\
\text { conceptual }\end{array}$ & $\begin{array}{l}\text { Tiempo de ciclo del } \\
\text { canal y nivel de in- } \\
\text { ventarios }\end{array}$ \\
\hline $\begin{array}{l}\text { Stock } \\
\text { et.al. } \\
(2000)\end{array}$ & $\begin{array}{l}\text { Falta de integración } \\
\text { entre logística y ca- } \\
\text { dena de suministro }\end{array}$ & EDI & $\begin{array}{l}\text { Logística Pro- } \\
\text { veedores y } \\
\text { clientes }\end{array}$ & $\begin{array}{l}\text { Estudio } \\
\text { empírico }\end{array}$ & $\begin{array}{l}\text { Rendimiento opera- } \\
\text { cional y rendimiento } \\
\text { financiero }\end{array}$ \\
\hline $\begin{array}{l}\text { Huisko- } \\
\text { nen y } \\
\text { Pirtrila } \\
(2002)\end{array}$ & $\begin{array}{l}\text { Necesidad de mejo- } \\
\text { rar la relación entre } \\
\text { Logística y clientes }\end{array}$ & $\begin{array}{l}\text { Intercambio de } \\
\text { información y tec- } \\
\text { nologías de infor- } \\
\text { mación }\end{array}$ & $\begin{array}{l}\text { Logística Pro- } \\
\text { veedores y } \\
\text { manufactura }\end{array}$ & $\begin{array}{c}\text { Estudio } \\
\text { conceptual }\end{array}$ & Buenas relaciones \\
\hline \multicolumn{6}{|c|}{ INVENTARIO } \\
\hline $\begin{array}{l}\text { Lu } \\
(1995) \\
\text { y Yao y } \\
\text { Chiou } \\
(2004)\end{array}$ & $\begin{array}{l}\text { Diferentes intervalos } \\
\text { de los pedidos }\end{array}$ & $\begin{array}{l}\text { Toma de decisio- } \\
\text { nes conjunta y } \\
\text { beneficios com- } \\
\text { partidos }\end{array}$ & $\begin{array}{l}\text { Un solo pro- } \\
\text { veedor- múlti- } \\
\text { ples compra- } \\
\text { dores }\end{array}$ & $\begin{array}{l}\text { Modelo } \\
\text { analítico }\end{array}$ & $\begin{array}{l}\text { Minimización de cos- } \\
\text { tos (ordenar, mante- } \\
\text { nimiento, compra) }\end{array}$ \\
\hline $\begin{array}{l}\text { Verwi- } \\
\text { jmeren } \\
\text { et.al. } \\
\text { (1996) }\end{array}$ & $\begin{array}{l}\text { Manejo independien- } \\
\text { te de los inventarios }\end{array}$ & $\begin{array}{l}\text { Tecnologías de in- } \\
\text { formación y bene- } \\
\text { ficios mutuos }\end{array}$ & $\begin{array}{l}\text { Red de la ca- } \\
\text { dena de sumi- } \\
\text { nistro }\end{array}$ & $\begin{array}{l}\text { Soluciones } \\
\text { de redes }\end{array}$ & $\begin{array}{l}\text { Mejora del servicio al } \\
\text { cliente. Aumento de } \\
\text { variedad de produc- } \\
\text { tos y bajos costos en } \\
\text { la cadena de sumi- } \\
\text { nistro }\end{array}$ \\
\hline $\begin{array}{l}\text { Moses y } \\
\text { Seshadri } \\
(2000)\end{array}$ & $\begin{array}{l}\text { Necesidad de ries- } \\
\text { go compartido. } \\
\text { Desajuste a nivel de } \\
\text { las existencias y el } \\
\text { período de revisión }\end{array}$ & $\begin{array}{l}\text { Toma de decisio- } \\
\text { nes conjunta y } \\
\text { descuentos por } \\
\text { cantidad }\end{array}$ & $\begin{array}{l}\text { Fabricante - } \\
\text { Minorista }\end{array}$ & Optimización & $\begin{array}{l}\text { Minimización de cos- } \\
\text { tos }\end{array}$ \\
\hline $\begin{array}{l}\text { Boyaci y } \\
\text { Gallego } \\
(2002)\end{array}$ & $\begin{array}{l}\text { Falta de coordinación } \\
\text { en las decisiones del } \\
\text { tamaño del lote y el } \\
\text { precio }\end{array}$ & $\begin{array}{l}\text { Plan conjunto de } \\
\text { precios y políticas } \\
\text { de reposición de } \\
\text { inventarios }\end{array}$ & $\begin{array}{l}\text { Un Mayorista } \\
\text { y múltiples } \\
\text { minoristas }\end{array}$ & $\begin{array}{l}\text { Problema } \\
\text { analítico de } \\
\text { optimización }\end{array}$ & $\begin{array}{l}\text { Maximizar las ganan- } \\
\text { cias de canal (venta } \\
\text { al por mayor precio } \\
\text { - inventario - gastos } \\
\text { relacionados) }\end{array}$ \\
\hline
\end{tabular}




\begin{tabular}{|c|c|c|c|c|c|}
\hline $\begin{array}{l}\text { Zhao } \\
\text { et.al. } \\
\text { (2004) }\end{array}$ & $\begin{array}{l}\text { Discrepancia en la } \\
\text { colocación de la or- } \\
\text { den }\end{array}$ & $\begin{array}{l}\text { Coordinación de } \\
\text { pedidos, intercam- } \\
\text { bio de la informa- } \\
\text { ción }\end{array}$ & $\begin{array}{l}\text { Un solo fabri- } \\
\text { cante múlti- } \\
\text { ples detallis- } \\
\text { tas }\end{array}$ & Simulación & $\begin{array}{l}\text { Minimización de cos- } \\
\text { tos y mejora del nivel } \\
\text { del servicio }\end{array}$ \\
\hline $\begin{array}{l}\text { Wu y } \\
\text { Ouyang } \\
(2003)\end{array}$ & $\begin{array}{l}\text { Independencia en } \\
\text { el cálculos de los } \\
\text { costos }\end{array}$ & $\begin{array}{l}\text { Consideración de } \\
\text { los costos conjun- } \\
\text { tos con escasez }\end{array}$ & $\begin{array}{l}\text { Vendedor - } \\
\text { comprador }\end{array}$ & $\begin{array}{l}\text { Modelo } \\
\text { analítico }\end{array}$ & $\begin{array}{l}\text { Minimización de cos- } \\
\text { tos }\end{array}$ \\
\hline $\begin{array}{l}\text { Zou et.al. } \\
\text { (2004) }\end{array}$ & $\begin{array}{l}\text { Diferentes tiempos } \\
\text { de procesamiento de } \\
\text { pedidos de los pro- } \\
\text { veedores y conflictos } \\
\text { en los incentivos }\end{array}$ & $\begin{array}{l}\text { Intercambio de } \\
\text { información y con- } \\
\text { tratos de reparto } \\
\text { de ingresos }\end{array}$ & $\begin{array}{l}\text { Múltiples pro- } \\
\text { veedores - un } \\
\text { solo fabrican- } \\
\text { te } 0 \text { ensam- } \\
\text { blador }\end{array}$ & $\begin{array}{l}\text { Modelo } \\
\text { analítico }\end{array}$ & Maximizar utilidades \\
\hline $\begin{array}{l}\text { Chen y } \\
\text { Chen } \\
(2005)\end{array}$ & $\begin{array}{l}\text { Necesidad de riesgo } \\
\text { compartido }\end{array}$ & $\begin{array}{l}\text { La consideración } \\
\text { conjunta de los } \\
\text { costos, el inter- } \\
\text { cambio de ahorro, } \\
\text { descuentos por } \\
\text { cantidad }\end{array}$ & $\begin{array}{l}\text { Fabricante - } \\
\text { detallista }\end{array}$ & Matemática & $\begin{array}{l}\text { Mejoramiento del } \\
\text { pareto }\end{array}$ \\
\hline $\begin{array}{l}\text { Piplani } \\
\text { y Fu } \\
\text { (2005) }\end{array}$ & $\begin{array}{l}\text { Decisiones desali- } \\
\text { neadas del inventario }\end{array}$ & $\begin{array}{l}\text { Reparto de costos } \\
\text { y contratos de ni- } \\
\text { vel de servicio }\end{array}$ & $\begin{array}{l}\text { Multi-eslabo- } \\
\text { nes }\end{array}$ & $\begin{array}{l}\text { Multi- } \\
\text { agentes } \\
\text { tecnológicos } \\
\text { y algoritmos } \\
\text { genéticos }\end{array}$ & $\begin{array}{l}\text { Minimización del } \\
\text { costo de manteni- } \\
\text { miento de inventario }\end{array}$ \\
\hline $\begin{array}{l}\text { Huq et.al. } \\
\text { (2006) }\end{array}$ & $\begin{array}{l}\text { Independencia en la } \\
\text { consideración de los } \\
\text { costos }\end{array}$ & $\begin{array}{l}\text { Consideración } \\
\text { conjunto de los } \\
\text { costos }\end{array}$ & $\begin{array}{l}\text { Multi- mayo- } \\
\text { ristas Multi- } \\
\text { detallistas }\end{array}$ & $\begin{array}{l}\text { Modelo } \\
\text { matemático y } \\
\text { simulación }\end{array}$ & $\begin{array}{l}\text { Minimización de los } \\
\text { costos de distribu- } \\
\text { ción y tiempos de } \\
\text { entrega }\end{array}$ \\
\hline $\begin{array}{l}\text { Barron } \\
(2007)\end{array}$ & $\begin{array}{l}\text { Diferentes tiempos } \\
\text { de ciclo }\end{array}$ & $\begin{array}{l}\text { Toma de decisio- } \\
\text { nes conjunta }\end{array}$ & $\begin{array}{l}\text { Cadena de } \\
\text { suministro } \\
\text { multieslabon }\end{array}$ & $\begin{array}{l}\text { Modelo } \\
\text { analítico }\end{array}$ & $\begin{array}{l}\text { Minimización de cos- } \\
\text { tos (ordenar y mante- } \\
\text { nimiento) }\end{array}$ \\
\hline \multicolumn{6}{|c|}{ PRONOSTICOS } \\
\hline $\begin{array}{l}\text { Aviv } \\
\text { (2001) }\end{array}$ & $\begin{array}{l}\text { La Toma de decisio- } \\
\text { nes independiente de } \\
\text { los pronósticos }\end{array}$ & $\begin{array}{l}\text { Toma de deci- } \\
\text { siones conjunta } \\
\text { e intercambio de } \\
\text { información sobre } \\
\text { la demanda }\end{array}$ & $\begin{array}{l}\text { Fabricante - } \\
\text { detallista }\end{array}$ & $\begin{array}{l}\text { Modelo } \\
\text { analítico }\end{array}$ & $\begin{array}{l}\text { Minimización de cos- } \\
\text { tos }\end{array}$ \\
\hline \multicolumn{6}{|c|}{ INVENTARIO - DISTRIBUCION } \\
\hline $\begin{array}{l}\text { Haq y } \\
\text { Kannan } \\
(2006)\end{array}$ & $\begin{array}{l}\text { Mentira potencial } \\
\text { en la reducción de } \\
\text { costos, considerando } \\
\text { todos los costos con- } \\
\text { juntamente }\end{array}$ & $\begin{array}{l}\text { La consideración } \\
\text { conjunta de los } \\
\text { costos en cada } \\
\text { nivel }\end{array}$ & Multi-eslabón & $\begin{array}{l}\text { Fuzzy AHP y } \\
\text { algoritmos } \\
\text { genéticos }\end{array}$ & $\begin{array}{l}\text { Minimización de } \\
\text { costos (manejo de in- } \\
\text { ventario, producción, } \\
\text { transporte) }\end{array}$ \\
\hline \multicolumn{6}{|c|}{ PRODUCCION - DISTRIBUCION } \\
\hline $\begin{array}{l}\text { Jaya- } \\
\text { raman } \\
\text { y Pirkul } \\
(2001)\end{array}$ & $\begin{array}{l}\text { La falta de integra- } \\
\text { ción en los diferentes } \\
\text { procesos de la cade- } \\
\text { na de suministro }\end{array}$ & $\begin{array}{l}\text { Minimización de } \\
\text { los costos conjun- } \\
\text { tos de producción } \\
\text { y distribución }\end{array}$ & Multi-eslabón & $\begin{array}{l}\text { Relajación } \\
\text { lagrangiana }\end{array}$ & $\begin{array}{l}\text { Minimización de cos- } \\
\text { tos (compras, pro- } \\
\text { ducción, distribución) }\end{array}$ \\
\hline
\end{tabular}




\begin{tabular}{|c|c|c|c|c|c|}
\hline $\begin{array}{l}\text { Pyke y } \\
\text { Cohen } \\
(1994)\end{array}$ & $\begin{array}{l}\text { Conflicto entre el ta- } \\
\text { maño de lote grande } \\
\text { (producción) y el ta- } \\
\text { maño de lote peque- } \\
\text { ño (distribución) }\end{array}$ & $\begin{array}{l}\text { Óptimos cercanos } \\
\text { de costo y niveles } \\
\text { de servicio, plan } \\
\text { conjunto }\end{array}$ & $\begin{array}{l}\text { Un fabricante } \\
\text { Un distribui- } \\
\text { dor } \\
\text { Un detallista }\end{array}$ & $\begin{array}{l}\text { Problema de } \\
\text { optimización } \\
\text { restringida }\end{array}$ & $\begin{array}{l}\text { Costos de producción } \\
\text { y niveles de servicio }\end{array}$ \\
\hline $\begin{array}{l}\text { Chandra } \\
\text { y Fischer } \\
\text { (1994) }\end{array}$ & $\begin{array}{l}\text { Costos de manejo de } \\
\text { inventario en múlti- } \\
\text { ples locaciones de la } \\
\text { cadena de suministro }\end{array}$ & $\begin{array}{l}\text { Coordinar la pro- } \\
\text { gramación de la } \\
\text { producción y de } \\
\text { rutas para vehí- } \\
\text { culos }\end{array}$ & Multi-eslabón & Heurísticas & $\begin{array}{l}\text { Minimización de cos- } \\
\text { tos (costos fijos de las } \\
\text { instalaciones, costos } \\
\text { de mantenimiento de } \\
\text { inventario, costos de } \\
\text { distribución) }\end{array}$ \\
\hline $\begin{array}{l}\text { Ganes } \\
\text { han } \\
(1999)\end{array}$ & $\begin{array}{l}\text { Falta de integración } \\
\text { en diferentes pro- } \\
\text { cesos }\end{array}$ & $\begin{array}{l}\text { Minimización de } \\
\text { los costos conjun- } \\
\text { tos de producción } \\
\text { y distribución }\end{array}$ & Multi-eslabón & $\begin{array}{l}\text { Progra- } \\
\text { mación } \\
\text { Matemática y } \\
\text { simulación }\end{array}$ & $\begin{array}{l}\text { Minimización de cos- } \\
\text { tos (compras, pro- } \\
\text { ducción, distribución) }\end{array}$ \\
\hline $\begin{array}{l}\text { Jang } \\
\text { et.al. } \\
\text { (2002) }\end{array}$ & $\begin{array}{l}\text { Necesidad de coor- } \\
\text { dinar producción y } \\
\text { distribución }\end{array}$ & $\begin{array}{l}\text { Minimización cos- } \\
\text { to conjunto }\end{array}$ & Multi-eslabón & $\begin{array}{l}\text { Heurísticas } \\
\text { lagrangianas } \\
\text { y algoritmos } \\
\text { genéticos }\end{array}$ & $\begin{array}{l}\text { Minimización de } \\
\text { costos (producción, } \\
\text { distribución) }\end{array}$ \\
\hline \multicolumn{6}{|c|}{ PRODUCCION - INVENTARIO } \\
\hline $\begin{array}{l}\text { Yang } \\
\text { y Wee } \\
(2002)\end{array}$ & $\begin{array}{l}\text { Conflicto en la bús- } \\
\text { queda de número de } \\
\text { entregas de un pedi- } \\
\text { do por el vendedor y } \\
\text { el comprador }\end{array}$ & $\begin{array}{l}\text { La toma de deci- } \\
\text { siones conjunta } \\
\text { y descuentos por } \\
\text { cantidad }\end{array}$ & $\begin{array}{l}\text { Un proveedor } \\
-\quad \text { múltiples } \\
\text { compradores }\end{array}$ & $\begin{array}{c}\text { Modelo } \\
\text { matemático }\end{array}$ & $\begin{array}{l}\text { Minimización de cos- } \\
\text { tos (mantenimiento y } \\
\text { de ordenar) }\end{array}$ \\
\hline $\begin{array}{l}\text { Hill y } \\
\text { Omar } \\
(2006)\end{array}$ & $\begin{array}{l}\text { Incremento de los } \\
\text { costos de almacena- } \\
\text { miento de inventario } \\
\text { en los sucesivos es- } \\
\text { labones de la cadena } \\
\text { de suministro }\end{array}$ & $\begin{array}{l}\text { Para diferentes } \\
\text { actores de la ca- } \\
\text { dena de suminis- } \\
\text { tro encontrar la } \\
\text { cantidad optima } \\
\text { de pedido y com- } \\
\text { partir beneficios }\end{array}$ & $\begin{array}{l}\text { Un proveedor } \\
\text { Un comprador }\end{array}$ & $\begin{array}{c}\text { Modelo } \\
\text { matemático }\end{array}$ & $\begin{array}{l}\text { Minimización de } \\
\text { costos (producción, } \\
\text { envío y almacena- } \\
\text { miento) }\end{array}$ \\
\hline $\begin{array}{l}\text { Hwarng } \\
\text { el al. } \\
(2005)\end{array}$ & $\begin{array}{l}\text { Gestión de la com- } \\
\text { plejidad }\end{array}$ & $\begin{array}{l}\text { Sincronización de } \\
\text { los ciclos de pro- } \\
\text { ducción y control } \\
\text { de riesgos }\end{array}$ & $\begin{array}{l}\text { Multi-eslabon } \\
\text { (5 niveles) }\end{array}$ & Simulación & $\begin{array}{l}\text { Nivel de existencias } \\
\text { promedio, la cartera } \\
\text { promedio y el costo } \\
\text { total promedio }\end{array}$ \\
\hline
\end{tabular}

Fuente: (Arshinder, 2008)

\section{3. ¿Qué tipos de integración existen?}

La literatura sugiere que hay dos formas interrelacionadas de tipos de integración que los fabricantes emplean regularmente, tal como se muestra en la Figura 2.

El primer tipo de integración incluye la coordinación e integración hacia adelante del flujo físico de entregas entre proveedores, fabricantes y clientes (Saunders, 1997), (Trento y Montczka, 1998). Otros han señalado la importancia de la integración de las entregas 


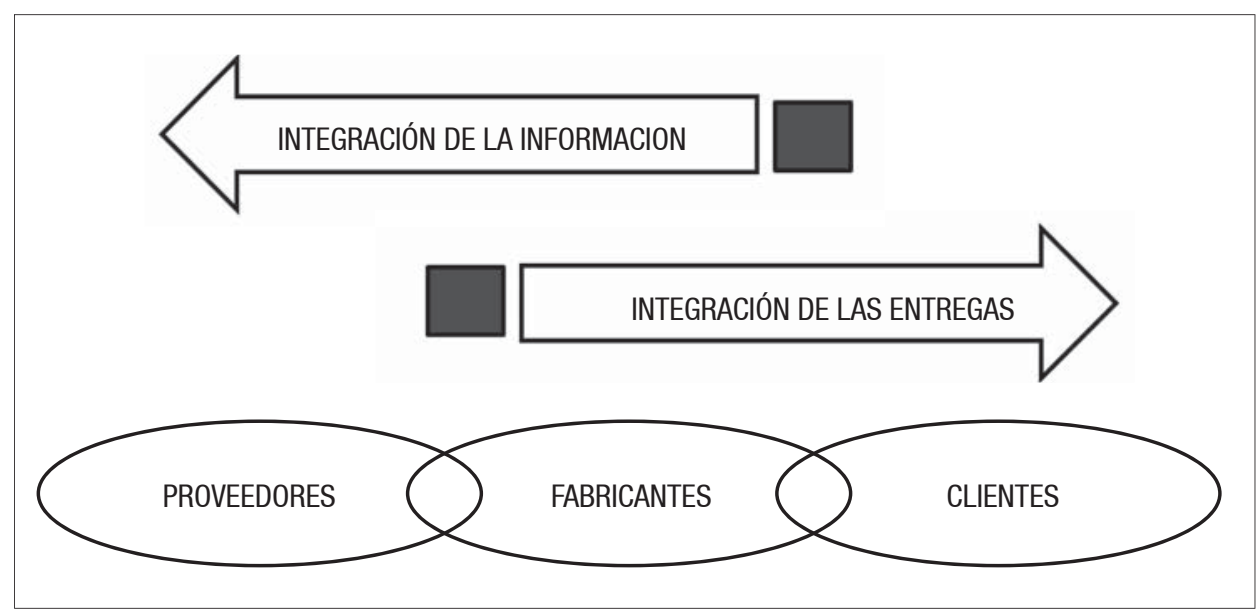

Figura 2. Integración de la cadena de suministro

en términos de implementar aplazamientos y masificación del producto en la cadena de suministro (Vernadat, 2002), (Ellinger, 1997), (Iain Dinwoodie, 2013). El otro tipo de integración prevalente incluye la coordinación hacia atrás de las tecnologías de información y el flujo de datos de los clientes a los proveedores (Vernadat, 2002), (Giuseppe Confessore, 2013).

Los flujos de información en esta dirección permitirán a las empresas responder directamente a las necesidades del cliente y puede ser coordinada a través de múltiples organizaciones o redes. Estos flujos de información fueron originalmente posibles mediante el uso del intercambio electrónico de datos (EDI). Esta tecnología ha sido sustituida por la Internet y la introducción del comercio electrónico (Marapoulos, 2005), (Tobias Schoenherra, 2012).

Las tecnologías de información permiten a múltiples organizaciones coordinar sus actividades en un esfuerzo para gestionar una verdadera cadena de suministro (Marapoulos, 2005), (Tobias Schoenherra, 2012), (Iain Dinwoodie, 2013), (Islam, 2013).

Vernadat, en su articulo "Enterprise modeling and integration", menciona algunos tipos de integración: Integración horizontal versus vertical, Integración intra-empresa versus Integración inter-empresa, Integración de sistemas versus integración de aplicaciones versus integración de negocios (Erhan Kutanoglu, 2008), (Kar, 2013) (Leung, 2013), (Daniel Prajogo, 2012), (Marcella De Martino, 2013).

\section{4. ¿Cómo se integra?}

En este apartado se contemplan específicamente a los mecanismos, técnicas y herramientas que hacen posible la integración en la cadena de suministro. 
Tabla IV. Taxonomía mecanismos de coordinación. Fuente: (Arshinder, 2008)

\begin{tabular}{|l|l|}
\hline Mecanismos de Coordinación & Autores \\
\hline $\begin{array}{l}\text { Contratos en la cadena de } \\
\text { Suministro }\end{array}$ & $\begin{array}{l}\text { Strank y Golsby (2000), Zou et.al.(2004), Piplani y Fu (2005), Hill y Omar (2006), } \\
\text { Yang y Wee (2002) }\end{array}$ \\
\hline Tecnologías de información & $\begin{array}{l}\text { Wilson (1995), Verwijimeren et.al.(1996), Lee et.al.(1999), Themistocles et.al. } \\
\text { (2004), Caglino et.al.(2005), Fin(2005), Liu et.al.(2005) }\end{array}$ \\
\hline Intercambio de información & $\begin{array}{l}\text { Zouet.al.(2004), Piplani y Fu(2005), Simchi-Levi et.al.(2000), Pyke et.al.(2000), } \\
\text { Simatupang y Sridharan(2002) }\end{array}$ \\
\hline Toma de decisiones conjunta & $\begin{array}{l}\text { Yang y Wee(2002), Lu(1995), Boyaci y Gallego(2002), Barron(2007), Haq y } \\
\text { Kannan(2006), Jayaraman y Pirkul(2001), Pyke y Cohen(1993), Chandra y } \\
\text { Fisher(1994), Ganeshan(1999), Jang et.al.(2002), Yang y Wee(2002), Hwarng } \\
\text { et.al.(2005) }\end{array}$ \\
\hline
\end{tabular}

\subsubsection{Mecanismos de Coordinación}

El uso de mecanismos de coordinación y su correcta aplicación, permiten mejorar el rendimiento de la cadena de suministro (Arshinder, 2007). Los problemas y conflictos en la coordinación de los actores de la cadena de suministro pueden ser resueltos a través de la aplicación de mecanismos de coordinación (Jayanth Jayaram, 2010),(Arshinder, 2008). Los mecanismos de coordinación pueden ser clasificados de acuerdo a la Tabla IV, así (Arshinder, 2008):

- El uso de los cuatro mecanismos de coordinación que a continuación se explican en detalle, permiten mejorar el rendimiento de la cadena de suministro (SC) y ayudar en la evaluación de la misma (Arshinder, 2008), (Flynn, 2010), (Yao, 2010).

- Los contratos en la cadena de suministro. Los miembros de la cadena de suministro coordinan el uso de contratos para mejorar la gestión de la relación proveedorcomprador. Los contratos especifican los parámetros (por ejemplo, cantidad, precio, tiempo y calidad) en el que un comprador realiza pedidos y un proveedor los cumple. Los objetivos de los contratos de la cadena de suministro son: aumentar el beneficio total de la cadena de suministro, reducir los costos, controlar el exceso o la falta de existencias y compartir los riesgos entre los socios de la cadena de suministro (Arshinder, 2008), (Barbara B. Flynn, 2010).

- Tecnología de la información. La tecnología de la información se utiliza para mejorar la coordinación entre organizaciones (McAfee, 2002; Sanders, 2008) y, a su vez, la coordinación entre organizaciones ha demostrado que tiene un impacto positivo en seleccionar las medidas de rendimiento de la empresa, tales como servicio al cliente, tiempo de entrega y costos de producción (Vickery et.al, 2003). Las tecnologías de información (TI) ayudan a conectar el punto de producción a la perfección con el punto de entrega o compra. Permite la planeación, el requerimiento y la estimación de los plazos de entrega con base en datos en tiempo real. Los avances en TI (por ejemplo INTERNET, EDI (intercambio electrónico de datos), ERP (Enterprise resource planning), E-businness (comercio electróni- 
co) y muchos más), permiten que las empresas puedan intercambiar rápidamente productos, información y utilizar métodos de colaboración para optimizar las operaciones en la cadena de suministro (Vernadat, 2002), (Pagell, 2004), (WookKim, 2009), (Mendoza, 2009), (Pineda, 2010), (Sanchez, 2005), (Sanchez, 2002), (Vernadat, 2002), (Jianming, 2010), (Pagell, 2004), (WookKim, 2009), (Mendoza, 2009), (Pineda, 2010), (Islam, 2013).

- El intercambio de información. Los miembros de la cadena de suministro coordinan el intercambio de información con respecto a la demanda, pedidos, inventarios, etc. El valor de intercambiar información incrementa el nivel de servicio del proveedor, reduciendo el ciclo de pedido. La política de intercambiar información produce reducciones en inventario y ahorro en costos (Yu et.al, 2001). Los datos de los puntos de venta ayudan al proveedor a anticipar mejor los futuros pedidos de los minoristas y reducen el efecto látigo (Arshinder, 2008), (Yao, 2010).

- Toma de decisiones conjunta. Las consideraciones conjuntas de reposiciones (Yao y Chou, 2004), costos de mantenimiento de inventario con demanda dinámica (Boctor et.al,2004), planeación colaborativa (Aviv, 2001), costos de procesos diferentes (Hag y kannan, 2006; Jayraman y Pirkue, 2001; Ganeshan, 1999), frecuencia de pedidos (Yang y Wee, 2002; Burson, 2007), tamaño de lote (Pyke y Cohen, 1993; Boyaer y Gallego, 2002), desarrollo de productos (Kim y Oh, 2005), se utilizan para mejorar el rendimiento de la cadena de suministro. Una toma de decisiones coherente ayuda a resolver conflictos entre los miembros de la cadena de suministro y en el manejo de excepciones en el caso de incertidumbre (Arshinder, 2008), (Mc laren, Head, \& Yufei, 2002), (Romano, 2003),(Petra Schebert, 2011), (Marcella De Martino, 2013) (Min Huang, 2013).

Otros mecanismos de integración a coordinar que se pueden considerar son: unificación de metas, precios especiales con respecto al tamaño del lote de producción o pedido, asignación de suministro de materia prima y producto terminado por el proveedor, reducción de tiempos ciclo en el abastecimiento, aplicación de políticas de descuento, aplicación de sistemas multimodales de transporte (Rakesh Nagi, 2009), (Pamela Danese, 2013) (Pantelis Longinidis, 2013).

\subsubsection{Técnicas de Integración}

Marco Perona y Nicola Saccani (2004), se refieren a las técnicas y herramientas de integración particularmente en relación a la gestión de las relaciones cliente-proveedor a través de la adopción de un conjunto de prácticas que apoyan los procesos de integración. Las técnicas de integración pueden ser definidas como las decisiones sobre cómo gestionar los procesos de la integración en la cadena de suministro (Marco Perona, 2004), (Piet A. Slats, 1995), (Ramanathan, 2013). En este sentido, las técnicas de integración se pueden agrupar en tres clases (Marco Perona, 2004):

- Las técnicas para la gestión de operaciones que sirven para coordinar la logística de los socios comerciales y procesos de manufactura. 
- Las técnicas para la gestión de la tecnología que sirven para coordinar e involucrar a los proveedores con los clientes en el desarrollo de procesos y actividades para nuevos productos.

- Las técnicas para planeación estratégica conjunta se dirigen a compartir la definición de objetivos de negocio y del mercado e implica directamente a la alta dirección de las empresas.

La Tabla V, proporciona una lista de técnicas de integración. A través de la adopción de técnicas de integración, las empresas pueden mejorar la eficiencia o la eficacia de los procesos de integración (Marco Perona, 2004), (Marapoulos, 2005).

\begin{tabular}{|c|c|c|c|}
\hline Dominio & Área & Técnica & Referencias \\
\hline \multirow{16}{*}{ Operaciones } & \multirow{4}{*}{ Reposición } & Justo a tiempo & Isaac (1985) \\
\hline & & Entregas frecuentes & Caputo et.al.(1996) \\
\hline & & Programa de reposición continua & Marien (2001) \\
\hline & & Inventario manejado por el vendedor (VMI) & James et.al.(1997) \\
\hline & \multirow{2}{*}{$\begin{array}{l}\text { Gestión coordinada } \\
\text { de materiales }\end{array}$} & Certificación de calidad & Manuali (1997) \\
\hline & & Paso libre de suministros & $\begin{array}{l}\text { De Toni y Nassimbeni } \\
\text { (1997) }\end{array}$ \\
\hline & \multirow{3}{*}{$\begin{array}{l}\text { Operaciones } \\
\text { coordinadas } \\
\text { de planeación } \\
\text { y control }\end{array}$} & Ordenes abiertas & Ferrozi et.al.(1993) \\
\hline & & Balanceo presupuestal & Jhonson (1999) \\
\hline & & $\begin{array}{l}\text { La planificación colaborativa, previsión y } \\
\text { reabastecimiento (CPFR) }\end{array}$ & White (2000) \\
\hline & \multirow{3}{*}{$\begin{array}{l}\text { Configuración } \\
\text { de la distribución } \\
\text { coordinada }\end{array}$} & Reconfiguración de la red de almacenes & Stank yHaut (1990) \\
\hline & & Reducción de canales & Magretta (1998) \\
\hline & & Colocación & Bartmess y Cerny (1992) \\
\hline & \multirow{4}{*}{$\begin{array}{l}\text { Gestión } \\
\text { de la distribución } \\
\text { coordinada }\end{array}$} & $\begin{array}{l}\text { Planeación de requerimientos de distribu- } \\
\text { ción (DRP) }\end{array}$ & Christopher (1992) \\
\hline & & DRP intercompañias & Novack et.al.(1993) \\
\hline & & Sistemas multi-pick y multi-drop & Caputo et.al.(1996) \\
\hline & & $\begin{array}{l}\text { Administración colaborativo del transporte } \\
\text { (CTM) }\end{array}$ & $\begin{array}{l}\text { Browning y White (2000), } \\
\text { Cooke }(2000)\end{array}$ \\
\hline
\end{tabular}




\begin{tabular}{|c|c|c|c|}
\hline \multirow{4}{*}{ Tecnología } & \multirow{2}{*}{ Rediseños conjuntos } & Procesos conjuntos de rediseño & Hewitt (1994) \\
\hline & & $\begin{array}{l}\text { Diseño y rediseño de producto para la ges- } \\
\text { tión de la cadena de suministro }\end{array}$ & Lee y Billington (1992) \\
\hline & \multirow{2}{*}{$\begin{array}{l}\text { Desarrollo de nuevos } \\
\text { productos }\end{array}$} & $\begin{array}{l}\text { Co-diseño } \\
\text { Ingeniería Virtual }\end{array}$ & $\begin{array}{l}\text { Maggiore y Dominioni } \\
\text { (1999) }\end{array}$ \\
\hline & & Innovación tecnológica conjunta & $\begin{array}{l}\text { Turnbull et.al.(1992), } \\
\text { De toni y Nassimbeni } \\
\text { (1997), Krause (1998), } \\
\text { Lazaric y Marengo (1997) }\end{array}$ \\
\hline \multirow{2}{*}{$\begin{array}{l}\text { Planeacion } \\
\text { estratégica }\end{array}$} & \multirow{2}{*}{$\begin{array}{l}\text { Coordinación de } \\
\text { planes estratégicos }\end{array}$} & Coordinación de negocios focalizados & Kaplan y Hurd (2002) \\
\hline & & $\begin{array}{l}\text { Coordinación de planes de expansión de } \\
\text { mercados }\end{array}$ & Magretta (1998) \\
\hline
\end{tabular}

\subsubsection{Herramientas de Integración}

Las herramientas de integración se pueden definir como los recursos y bienes dedicados a apoyar o permitir la adopción de una o más técnicas. Las herramientas han sido agrupadas en tres clases (Marco Perona, 2004), (Barbara B. Flynn, 2010), (Wantao Yu, 2013):

- Herramientas de información que mejoran la eficiencia y eficacia del intercambio de información en la gestión de operaciones, logística y desarrollo de nuevos productos.

- Herramientas administrativas, se utilizan para planear, medir, controlar e incentivar la ejecución de los procesos de integración.

- Herramientas organizativas, tienen el propósito de mejorar el rendimiento de los procesos de integración en los casos de interacción compleja.

La Tabla VI, muestra una lista de las herramientas de integración (Marco Perona, 2004).

\section{5. ¿Para qué se integra?}

Hay varias razones, entre las cuales podemos mencionar las siguientes (Damien, 2005), (Arshinder, 2008), (Laureano Jimenez, 2006), (Themistocleus, 2004), (Katrina Lintukangas, 2009), (Pamela Danese, 2013) (Pantelis Longinidis, 2013):

- Uno de los grandes propósitos de la integración de la información es llevar a cabo la transmisión en tiempo real y procesamiento de la información necesaria para la toma de decisiones en la cadena de suministro. 
Tabla VI. Taxonomía Herramientas de integración. Fuente: (Marco Perona, 2004)

\begin{tabular}{|c|c|c|c|}
\hline Dominio & Área & Herramienta & Referencia \\
\hline \multirow{12}{*}{ 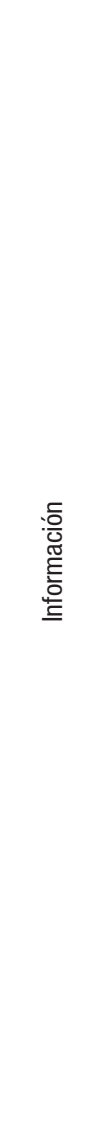 } & \multirow{3}{*}{$\begin{array}{l}\text { Contratación } \\
\text { electrónica }\end{array}$} & Intercambio de datos electrónicos (EDI) & $\begin{array}{l}\text { Banerjee y Sriram } \\
\text { (1995), Ramamurthy } \\
\text { et.al. (1999) }\end{array}$ \\
\hline & & Mercados electrónicos (E-marketplaces) & Varda (1999) \\
\hline & & $\begin{array}{l}\text { Internet basados en asociaciones interac- } \\
\text { tivas (I-BIP) }\end{array}$ & $\begin{array}{l}\text { Ovum(1999) } \\
\text { Economist (1999) } \\
\text { Kehoe y Boughton } \\
(2001)\end{array}$ \\
\hline & \multirow{4}{*}{$\begin{array}{l}\text { Integración } \\
\text { de la información }\end{array}$} & $\begin{array}{l}\text { Producción integrada y bases de datos de } \\
\text { inventario }\end{array}$ & $\begin{array}{l}\text { Hall (1997) } \\
\text { Lee y Billington (1992) }\end{array}$ \\
\hline & & Programas integrados DRP & Lee y Whang (2001) \\
\hline & & Datos integrados de ingeniería & Hall (1997) \\
\hline & & $\begin{array}{l}\text { Administración (EDM)/producto } \\
\text { Manejo de datos(PDM) }\end{array}$ & Marcial et.al. (1997) \\
\hline & \multirow{2}{*}{$\begin{array}{l}\text { Sistemas de } \\
\text { monitoreo electrónico }\end{array}$} & $\begin{array}{l}\text { Sistemas de monitoreo a las entregas y } \\
\text { rastreo de los productos }\end{array}$ & $\begin{array}{l}\text { Viotti (1997) } \\
\text { Manualli (1997) }\end{array}$ \\
\hline & & Sistemas de identificación automática & Lee y Whang (2001) \\
\hline & \multirow{3}{*}{$\begin{array}{l}\text { Aplicaciones } \\
\text { de software } \\
\text { colaborativo }\end{array}$} & $\begin{array}{l}\text { Trabajo cooperativo soportado por com- } \\
\text { putadora (CSCW) }\end{array}$ & $\begin{array}{l}\text { Luzack y Eversheim } \\
\text { (1999) }\end{array}$ \\
\hline & & $\begin{array}{l}\text { Diseño integrado asistido por computador } \\
\text { (CAD) }\end{array}$ & $\begin{array}{l}\text { Luzack y Eversheim } \\
\text { (1999) }\end{array}$ \\
\hline & & $\begin{array}{l}\text { Manufactura integrada asistida por com- } \\
\text { putador (CAM) }\end{array}$ & $\begin{array}{l}\text { Luzack y Eversheim } \\
\text { (1999) }\end{array}$ \\
\hline \multirow{5}{*}{ 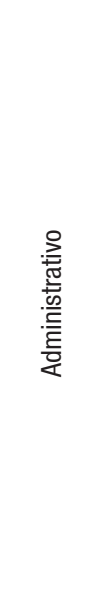 } & \multirow{2}{*}{$\begin{array}{l}\text { Sistemas de } \\
\text { evaluación } \\
\text { de proveedores }\end{array}$} & $\begin{array}{l}\text { Sistemas de selección de proveedores } \\
\text { (VSS) }\end{array}$ & $\begin{array}{l}\text { Masella y Rangone } \\
(2000)\end{array}$ \\
\hline & & $\begin{array}{l}\text { Sistemas de calificación de proveedores } \\
\text { (VRS) }\end{array}$ & James et.al. (1997) \\
\hline & $\begin{array}{l}\text { Sistemas de contabilidad de } \\
\text { costos en la cadena de su- } \\
\text { ministro }\end{array}$ & $\begin{array}{l}\text { Gestión de costos interorganizacional } \\
\text { Sistema de costos kaizen }\end{array}$ & Slagmulder (2001) \\
\hline & $\begin{array}{l}\text { Indicadores de desempeño } \\
\text { de la cadena de suministro }\end{array}$ & $\begin{array}{l}\text { Sistemas de medición del desempeño de } \\
\text { la cadena de suministro }\end{array}$ & $\begin{array}{l}\text { De Toni y } \\
\text { (2001) } \\
\text { Gunchia } \\
(2001)\end{array}$ \\
\hline & $\begin{array}{l}\text { Sistemas de incentivos en la } \\
\text { cadena de suministro }\end{array}$ & $\begin{array}{l}\text { Sistemas de incentivos en las empresas } \\
\text { Contratos }\end{array}$ & $\begin{array}{l}\text { Lee y Whang (2001) } \\
\text { Voss y Schneidereit } \\
\text { (2001) }\end{array}$ \\
\hline
\end{tabular}




\begin{tabular}{|l|l|l|l|}
\hline \multirow{2}{*}{} & Roles de las interfaces & Ingenieros residentes & Caputo y Zirpoli (2001) \\
\cline { 3 - 4 } & & Interfases con los directores & Cooper et.al. (1997) \\
\cline { 3 - 4 } & & $\begin{array}{l}\text { Alianza direccionada a las funciones ge- } \\
\text { renciales }\end{array}$ & Dyer et.al. (2001) \\
\cline { 3 - 5 } & $\begin{array}{l}\text { Unidades organizativas en } \\
\text { las empresas }\end{array}$ & $\begin{array}{l}\text { Equipos para el desarrollo de nuevos pro- } \\
\text { ductos }\end{array}$ & Eversheim (1996) \\
\cline { 2 - 4 } & & Equipos de mejora de producto-proceso & $\begin{array}{l}\text { Haug (1993) } \\
\text { Handfield et.al.(2000) }\end{array}$ \\
\hline
\end{tabular}

- Se ha argumentado que contar con una integración logística sólida, reducirá diversos problemas, tales como el efecto látigo.

- En general, la integración logística (Integración de flujo de bienes) permite a las empresas y sus socios de la cadena de suministro actuar como una sola entidad que se traduce en un mejor desempeño a través de toda la cadena.

- De la mejora de la integración logística entre los socios de la cadena de suministro, se obtiene un número de ventajas operativas, incluido la reducción de costos (Noteboom, 1992), reducción de los plazos de entrega (Lee et.al, 2005), reducción de los riesgos (Clemens et.al, 1993), así como la mejora en las ventas, mejora en la distribución, servicio al cliente y niveles de servicio.

- La mayoría de los estudios empíricos sobre la integración de la cadena de suministro muestran una relación positiva entre la integración y el rendimiento (Van der Vaart y Van Donk, 2008). De Toni y Nassenbern (1999) encontraron que las plantas que tienen mejores rendimientos exhiben un alto nivel de interacciones logísticas, Frohlich y Wesbrook (2001) encontraron que entre más amplio sea el arco de integración, mejora el rendimiento, Sheu et.al.(2006) encontró que altos niveles de colaboración resultan en eficiencias operacionales en la cadena de suministro y finalmente Li et.al (2009) encontró que la integración de la cadena de suministro está significativamente relacionada con el rendimiento de la cadena.

- El objetivo de la integración de la cadena de suministro es lograr el flujo efectivo y eficiente de productos y servicios, información, dinero y decisiones para proporcionar el máximo valor al cliente a bajo costo y alta velocidad.

- La falta de coordinación en la cadena de suministro puede resultar en un rendimiento pobre de la cadena de suministro.

- Las consecuencias de la falta de coordinación son: pronósticos inexactos, baja utilización de la capacidad, exceso de inventarios, servicio al cliente inadecuado, tiempo en el mercado, tiempo de respuesta al cumplimiento de las ordenes, calidad, atención al cliente y satisfacción del cliente.

- Existen múltiples beneficios derivados de una efectiva coordinación de la cadena de suministro (SCC). Algunos de estos beneficios incluyen: la eliminación del exceso de inventario, reducción de los plazos de entrega, aumento de las ventas, servicio al cliente, desarrollo eficiente de productos, bajos costos de fabricación, 
una mayor flexibilidad para hacer frente a la incertidumbre de alta demanda, una mayor retención de clientes y mejoras en los ingresos (Fish et.al., 1994; Lee et.al., 1997; Horvath 2001). La coordinación se percibe como un requisito previo para integrar las operaciones de las entidades de la cadena de suministro para alcanzar objetivos comunes.

La Tabla VII ilustra cómo las organizaciones integran tres tipos de sistemas (Custom, Packaged y e-business solutions) que se permutan entre sí generando otros sistemas. Sobre la base de estas permutaciones, los autores definen las clasificaciones de los tipos de sistemas que se integran en la cadena de suministro (Themistocleus, 2004).

\section{6 ¿Qué estrategias de integración existen?}

En la última década ha habido un creciente consenso sobre la importancia estratégica de la integración de proveedores, fabricantes y clientes (Lummus et.al, 2001; Heeketal Van, 2001; Barrat, 2004). Las estrategias de integración se pueden clasificar en tres grupos: estratégicas, tecnológicas y operativas. Entre las diversas estrategias de integración, se mencionan las siguientes (Piet A. Slats, 1995), (Yuanqiong, 2012), (WookKim, 2009), (Swierczek, 2013), (Wantao Yu, 2013):

A nivel operativo las estrategias se clasifican en:

- Coordinación cliente-proveedor

- Coordinación producción-distribución

- Coordinación distribución-inventarios

A nivel estratégico, las estrategias se orientan en:

- Coordinación de la planeación estratégica

- Coordinación del enfoque de negocios

A nivel tecnológico, las estrategias se orientan en:

- Rediseño conjunto de procesos

- Desarrollo conjunto de nuevos productos

- Ingeniería virtual

- Innovación tecnológica conjunta

Entre las estrategias relacionadas con la coordinación de la gestión de materiales (a nivel operativo) se pueden mencionar entre otras:

- Adquisiciones dictaminadas

- Ordenes globales

- Dimensionamiento conjunto de la capacidad de producción

- Colaboración en la planeación de pronostico y suministro 
Tabla VII. Taxonomía de los diferentes tipos de sistemas que son integrados

\begin{tabular}{|l|l|c|}
\hline \multicolumn{1}{|c|}{$\begin{array}{c}\text { Clasificacion de } \\
\text { tipos de sistemas }\end{array}$} & \multicolumn{1}{c}{ Descripcion } \\
\hline Integración & $\begin{array}{l}\text { Dentro de la organización EAl requiere la integración de aplicaciones interempresariales } \\
\text { e intraempresariales. Al incorporar todos los sistemas requeridos, muchas aplicaciones } \\
\text { personalizadas como aplicaciones heredadas y bases de datos están integrados en una } \\
\text { infraestructura común, para automatizar totalmente los procesos de negocio. Como } \\
\text { los sistemas personalizados no se han desarrollado para colaborar con otros siste- } \\
\text { mas, tienen puntos de acceso limitado a la integración. En concreto, bases de datos } \\
\text { e interfaces de usuario son los únicos puntos posibles de integración en la mayoría } \\
\text { de las aplicaciones personalizadas. Un caso típico de esta clasificación podría ser la } \\
\text { incorporación de sistemas heredados que tienen que ver con la gestión de promociones } \\
\text { (por ejemplo, existencias, cuentas de proveedores). En este caso, datos de las bases } \\
\text { de datos e interfaces de usuario debe ser extraído y enviado de una organización (por } \\
\text { ejemplo, minorista) a otra (por ejemplo, proveedor). Por lo tanto, las tecnologías que } \\
\text { extraen datos de una base de datos o en una pantalla son necesarias para apoyar esta } \\
\text { clasificación de los sistemas. }\end{array}$
\end{tabular}

Integración

Custom-to-packaged
Este es un enfoque común cuando las organizaciones adoptan EAl ya que las aplicaciones empaquetadas como los sistemas ERP en muchos casos han fracasado en lograr la integración y co-existir junto a las aplicaciones personalizadas. Un caso típico de este tipo podría ser la integración de un sistema heredado que se ocupa de la producción, y un módulo de ERP que se encarga de los pedidos de clientes o proveedores. Si bien, los sistemas ERP no fueron diseñados para incorporar otras aplicaciones autónomas, una diversidad de técnicas de enfoques y herramientas se pueden utilizar para lograr la integración entre los sistemas ERP y aplicaciones dispares. Desde una perspectiva técnica, la incorporación de sistemas ERP se puede lograr en los distintos niveles de datos, incluyendo objetos / componentes, como a nivel de interfaces.

Integración

Custom-to-e-business

Muchas soluciones e-business requieren una estrecha colaboración con las aplicaciones antiguas para apoyar los procesos y tareas de los e-business habilitados. Como resultado, las aplicaciones personalizadas (por ejemplo, stocks) se incorporan con los sistemas de e-business para integrar y automatizar los procesos inter-organizacionales de negocios. Del mismo modo, en muchos casos, la funcionalidad de una solución ebusiness se utiliza para apoyar los sistemas personalizados. Por ejemplo, una e-store actualiza un sistema personalizado que tiene que ver con la disponibilidad de stock. La información proporcionada por la solución de e-business es fundamental no sólo para la funcionalidad de la aplicación de valores, sino también para la cadena de suministro, ya que soporta la automatización y la integración de procesos de negocio específicos. Las tecnologías que apoyan la incorporación y el intercambio de datos, objetos e interfaces son necesarias para apoyar esta clasificación de los sistemas.
Integración

Packaged-to-packaged
En este caso, diferentes sistemas empaquetados, tal como las diferentes versiones de un sistema ERP o diferentes módulos de ERP que existen en una organización se unifican en una infraestructura común integrada. APIs son proporcionados por los sistemas ERP para permitir que otras aplicaciones puedan acceder a la funcionalidad ERP 0 de datos. Datos, mensajes y objetos se pueden introducir o transferir a un sistema ERP a través de APIs. Por lo tanto, la incorporación de un sistema empaquetado a otro sistema empaquetado requiere APIs así como también las tecnologías que soportan la extracción y transmisión de datos, mensajes y objetos. 


\begin{tabular}{|l|l|}
\hline Integración Packaged- & $\begin{array}{l}\text { Las organizaciones aprovechan la tecnología EAl y el comercio electrónico cuando integran } \\
\text { sus soluciones e-business con paquetes de aplicaciones como sistemas ERP que pueden } \\
\text { ser usados como sistema de back-office para apoyar la funcionalidad de e-business (aplica- } \\
\text { ciones front-end). En este caso, los procesos que tienen que ver con e-sale, e-procurement } \\
\text { y e-supply chain se integran con los sistemas empaquetados. Aplicaciones de negocio } \\
\text { electrónico a menudo se basan en tecnologías de objetos distribuidos (DOT) (por ejemplo, } \\
\text { Enterprise Java Beans, CORBA, DCOM / COM) y/o lenguajes estándar orientados a Internet } \\
\text { (por ejemplo, XML, HTML). Por lo tanto, las tecnologías que integran los datos, los objetos, } \\
\text { las interfaces y los mensajes son importantes para la integración de los sistemas empaque- } \\
\text { tados y la integración de e-business. }\end{array}$ \\
\hline $\begin{array}{l}\text { Integración Ebusiness-to- } \\
\text { ebusiness }\end{array}$ & $\begin{array}{l}\text { En este enfoque, una aplicación de e-business está integrado y es compatible con la fun- } \\
\text { cionalidad de otra solución de e-business. Por ejemplo, un punto de venta electrónico es } \\
\text { incorporado con e-supply chain para compartir datos que son importantes para la aplicación } \\
\text { de esta última (por ejemplo, pedidos de clientes, datos de cliente, etc.) La integración de } \\
\text { aplicaciones e-business puede ser facilitado por las tecnologías basadas en mensajes (por } \\
\text { ejemplo, XML), distribuidos tecnologías de objetos (por ejemplo, CORBA) y tecnologías de } \\
\text { bases de datos orientados (Java Database Connectivity, JDBC). }\end{array}$ \\
\hline $\begin{array}{l}\text { Integración } \\
\text { to-packaged-to-e- } \\
\text { business }\end{array}$ & $\begin{array}{l}\text { Este enfoque se centra en el desarrollo de una infraestructura integrada que integra los } \\
\text { procesos y aplicaciones en los departamentos, empresas o a nivel de toda la empresa. Los } \\
\text { tipos de sistemas que se incorporan requieren tecnologías de integración que soportan } \\
\text { todos los niveles de integración. Por lo tanto, las tecnologías que faciliten datos, objetos, } \\
\text { interfaces y mensajes son requeridas. }\end{array}$ \\
\hline
\end{tabular}

Fuente: (Themistocleus, 2004).

Las estrategias más comunes para el diseño de la red de distribución (a nivel estratégico) son:

- La reconfiguración de la red de almacenes

- Acortamiento del canal de comercialización

- Localización.

Las estrategias de gestión de distribución (a nivel operativo) incluyen:

- Coordinación de la planeación de los requerimientos de distribución entre compañías.

- Sistemas multiloteo (multi-pick)

- Multientregas (Multi-drop)

- Esquemas de gestión de transporte colaborativo.

Dentro de las estrategias de coordinación para la gestión de inventarios (a nivel operativo), podemos mencionar:

- Estrategia de desarrollo conjunto de ordenes (DCO)

- Estrategias justo a tiempo

- Respuesta rápida (QR: Quick Response)

- Estrategia de reaprovisionamiento eficiente (ER)

- Reaprovisionamiento continuo (CR) 
- Planeación, Pronostico y reabastecimiento (CPFR)

- Inventario administrado por el proveedor (VMI)

- Estrategia gestión de disponibilidad por el proveedor (SMA)

- Estrategia épocas comunes de resurtido (CRE)

\section{7 ¿Cómo se mide la integración?}

Muchos autores parecen estar de acuerdo con que un elevado nivel de integración tiene un impacto positivo sobre los resultados de las empresas pertenecientes a la cadena de suministro. Mientras algunos autores han aportado evidencia empírica de esta afirmación (Frohlich y Westbrook, 2001; Droge et.al., 2004; Gimenez y Ventura, 2005), otros tienen dudas y sugieren que esta relación es más retórica que real (Van der Vaart y Van Donk, 2008; Fabbe-Costes y Jahre,2007) ya que existe poco consenso en cómo capturar la esencia de la integración y que los tipos de resultados sobre los que se mide su influencia varían de unos autores a otros (Romano, 2001), (Frolich, 2001), (Paul Cousins, 2006), (WookKim, 2009), (Qiu Hong Zhao S. C., 2010), (Yan Cui, 2013), (Ahmed Musa, 2013).

En la literatura, para medir la integración se proponen diversos indicadores: la intensidad de la comunicación, la coordinación de actividades, la creación de equipos, la ausencia de fronteras rígidas entre las actividades logísticas de las empresas y las de los proveedores o clientes, la elaboración de decisiones conjuntas o el intercambio de información (Thomas y Griffin, 1996; O’Leary-Kelly y Flores, 2002). Pero, ¿por qué las empresas realizan estos cambios en su estructura y en su relación con otros agentes de la cadena de suministro? (Frolich, 2001), (Paul Cousins, 2006), (WookKim, 2009), (Nagurney, 2009), (Devendra Choudhary, 2013).

La respuesta es que existe un consenso de que a mayor grado (intensidad) de integración, la empresa va a obtener una ventaja competitiva y con ella, unos mejores resultados. Los resultados logísticos más estudiados han sido la eficiencia en costes, la fiabilidad de entrega y la flexibilidad. En primer lugar, la integración logística supondrá para la empresa un ahorro en costos por varios motivos (Uk Mike, 1996), (Frolich, 2001), (Marc, 2002), (Paul Cousins, 2006), (Taco Van Der Vaart, 2008), (WookKim, 2009), (Cornelia Droge, 2012), (Danese, 2013), (Islam, 2013):

- La reducción de inventario, ya que la relación con proveedores hace posible acuerdos de entrega de materias primas que favorecerán la reducción del stock,

- La disminución de los costes de transacción y de la incertidumbre de previsión de la demanda y del aprovisionamiento y

- Una mejor coordinación y cooperación entre las áreas funcionales y entre las empresas participantes.

En segundo lugar mejora la fiabilidad de entrega, es decir, la capacidad que tiene una organización de proveer a tiempo el tipo y la cantidad de producto requerido por 
los clientes en cada momento (Frolich, 2001), (Paul Cousins, 2006), (WookKim, 2009), (Giuseppe Confessore, 2013).

En tercer lugar, aumenta la flexibilidad, que es la capacidad del sistema logístico para comunicarse con los clientes y ajustar los esquemas de producción y distribución según las necesidades particulares de los mismos (Frolich, 2001), (Paul Cousins, 2006), (WookKim, 2009), (Chee Yew Wonga, 2011).

Frohlich y Westbrook (2001) dieron respuesta a esas cuestiones (intensidad y dirección de la integración) mediante un instrumento denominado arcos de integración donde se muestra cómo las empresas toman decisiones estratégicas respecto a la amplitud deseada de su integración y la dirección de la misma: aguas arriba o aguas abajo. Los distintos arcos de integración se construyen siguiendo las instrucciones que aparecen en la Figura 3 (Frolich, 2001), (GunaseKaran, 2004), (Muñoz, 2006), (Yuliang Yaoa, 2007), (Xiande Zhao, 2008), (Gang Li, 2009), (Bernd Sholz-Reiter, 2010), (Danese, 2013).

Como se observa en la Figura 3, algunos fabricantes deciden participar relativamente poco con proveedores y clientes y así tener un arco relativamente estrecho de integración. Otros fabricantes, al contrario, aplican una estrategia con un amplio arco de integración, es decir, integran ampliamente sus organizaciones con los proveedores y los clientes. Es evidente que a un mayor nivel de integración entre proveedores y clientes de la cadena de suministro, mayores serán los beneficios (Stevens, 1989; Lee et.al, 1997; Metters, 1997; Naransinham y Jayaran, 1998; Anderson y Katz, 1998; Hines et.al, 1998; Jhonson, 1999). Tan et.al (1998), señalaron que cuando las empresas se integran y actúan como una entidad, el rendimiento se mejora en toda la cadena. Handfield y Nichols (1999), argumentaron que ahora los fabricantes no solo deben gestionar sus propias organizaciones sino también estar involucrados en la gestión de la red, tanto en los flujos de información como en los flujos de bienes físicos a través de la cadena de suministro (Frolich, 2001).

El nivel de integración se mide a través del análisis factorial sobre actividades de colaboración entre la empresa y sus clientes y proveedores. La puntuación factorial obtenida por cada empresa (método de regresión) sirve para clasificarla en el cuartil superior (superior al 75\%), inferior (inferior al 25\%) o intermedio. Así se definen cinco grupos, excluyentes entre sí: empresas con bajos niveles de integración con proveedores y clientes (arco interno), aquellas con niveles intermedios (arco periférico) y aquellas con niveles elevados (arco externo). Cuando hay asimetría y se está integrado con un miembro del canal y no con el otro, da lugar al arco hacia el proveedor y el arco hacia el cliente. Las conclusiones a las que llegaron Frohlich y Westbrook (2001) fueron que a mayor amplitud del arco, mejores resultados y que si el arco era asimétrico los resultados no eran mejores que si no había integración (Marapoulos, 2005), (Sabine Fliess, 2006), (Qiu Hong Zhao S. C., 2010), (Salih Zeki, 2011), (Tobias Schoenherra, 2012), (Yuanqiong, 2012), (Farahani, 2013). 


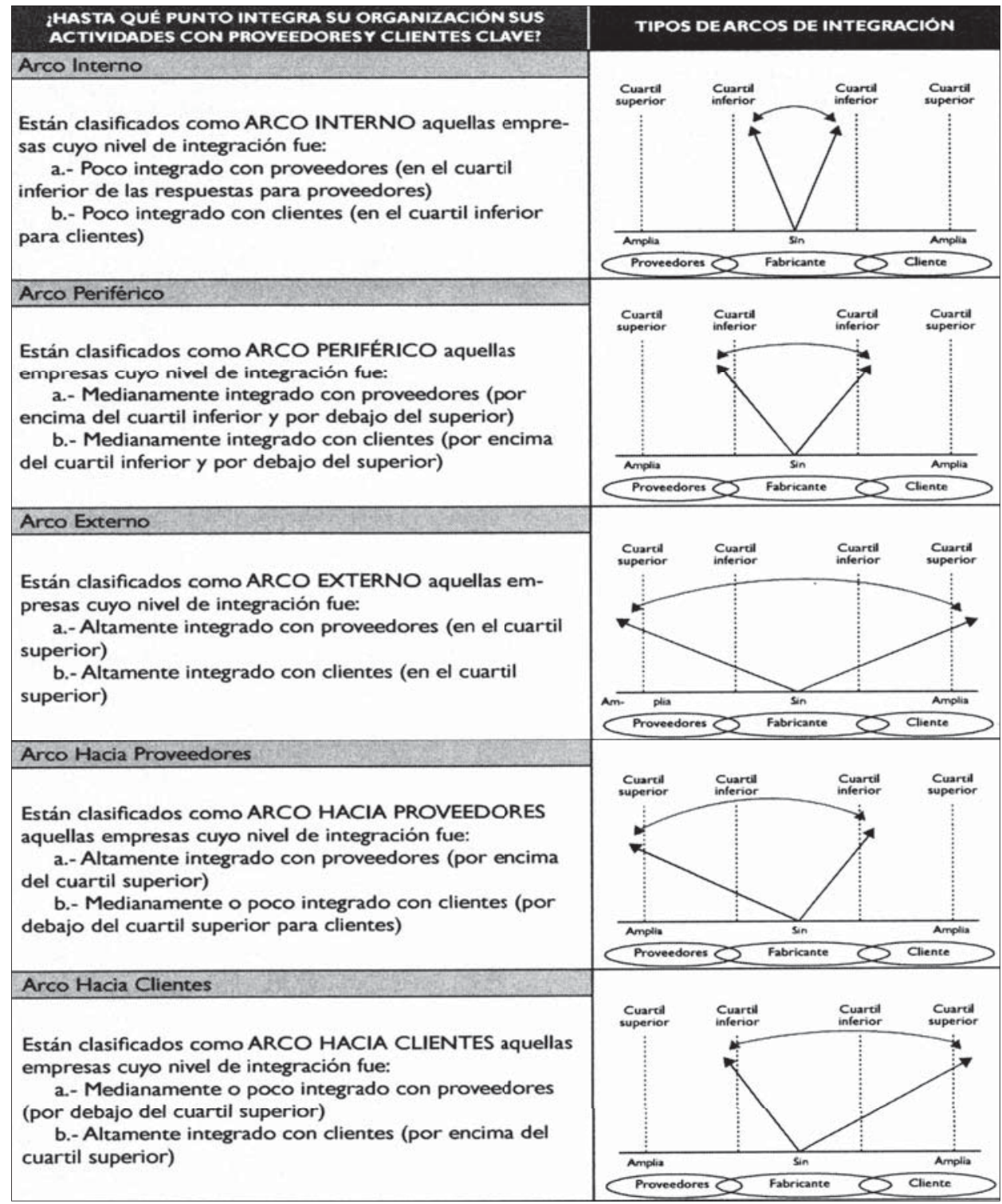

Figura 3. Arcos de Integración. Fuente: (Frolich, 2001)

La relación de indicadores de desempeño adecuados es un reto, debido a la complejidad inherente y la interdependencia de las cadenas de suministro. Mientras Chen y Paulraj (2004) sostiene que el desempeño financiero debe ser el criterio principal de valoración del desempeño de la cadena de suministro, otros han descrito las limitaciones de confiar únicamente en las medidas financieras de rendimiento (Dixon et.al, 1990, Eccles y Burn, 1992, Hall, 1993. Jhonson y Kaplan, 1987; Skinner, 1971). Van Hock (1998) y Beamon (1999) sugirieron que la medición del desempeño de la cadena de suministro 
debe incluir indicadores operacionales, tales como servicio al cliente y la capacidad de responder a un entorno cambiante. Nelly et.al (1995) enumeran costo, tiempo, calidad, entrega y flexibilidad como medidas importantes del rendimiento operacional (Themistocleus, 2004), (Indranil Bose, 2008), (Christos Maravelias, 2009), (Burcu Keskin, 2010), (Jiang Yanhuia, 2011), (C.F. Cheung, 2012), (Devendra Choudhary, 2013).

De otro lado, los principales indicadores que caracterizan la competitividad del sistema logístico y los niveles que en ellos muestran las empresas líderes en países desarrollados como Japón, Francia, Estados Unidos o Alemania son (Acero E, 2012), (Edrisi Muñoz, 2013), (Farahani, 2013):

- Inventario promedio/Ventas $=20,0 \%$

- Costo logístico/Ventas $=5,1 \%$

- Oportunidad en los aprovisionamientos $=90,0 \%$

- Oportunidad en los suministros a los clientes contra pedidos $=90,0 \%$

- Oportunidad en los suministros a los clientes contra almacén $=88,0 \%$

- Suministros perfectos de los proveedores $=89,5 \%$

- Suministros de pedidos perfectos a los clientes $=95,0 \%$

- Utilización de las capacidades de producción = 80,0\%

- Utilización de las capacidades de almacenaje $=90,0 \%$

- Cobertura del inventario de productos terminados $=21,0$ días

- Cobertura del inventario de materia prima y materiales $=43,5$ días

Las empresas tienen definido un sistema de indicadores para caracterizar y evaluar la gestión logística en general y en cada una de las actividades y unidades que conforman el sistema logístico de la organización, por otra parte, realizan sistemáticamente Benchmarking con las empresas que exhiben resultados de avanzada en las distintas actividades logísticas. Además, tiene adecuadamente organizado todos los registros de dichos indicadores. Dichos registros sirven de fundamento para una activa gestión de mejora del servicio al cliente sobre la base de lograr una adecuada diferenciación de los clientes. En última instancia, la efectividad de la gestión del sistema logístico tiene como fin elevar metódicamente la competitividad de la empresa. En tal caso la empresa exhibe el impacto del sistema logístico en un crecimiento sostenido de la competitividad de la compañía, la cual se refleja en la satisfacción sistemática de los siguientes indicadores (Diana Ballesteros, 2004), (Giuseppe Confessore, 2013), (Islam, 2013):

- Ritmo de crecimiento de los ingresos anuales

- Un ritmo de crecimiento de la ganancia superior al ritmo de crecimiento de los ingresos

- Crecimiento del mercado objetivo, incluyendo el aumento de las exportaciones

- Aumento de la cuota de mercado

- Crecimiento de la productividad y del salario medio. 


\section{Conclusiones}

En términos generales, la integración de toma de decisiones en la red logística requiere tiempo, recursos, inversión, infraestructura, apropiación de tecnologías de información para ser abordada en forma efectiva. En cuanto a las tecnologías de información, es importante resaltar que requieren ajustes e integración con otras plataformas, específicamente el software desarrollado como ERP. Esto hace que la implementación ERP sea compleja y requiera el desarrollo de sistemas de información para mejorar los procesos de integración.

En la revisión realizada se evidenció que aún cuando se han desarrollado e implementado procesos de integración con resultados positivos, no obstante todavía falta por mejorar la integración de la toma de decisiones en la red logística y para ello se siguen desarrollado modelos matemáticos, software, sistemas de información, estrategias de integración para mejorar aun mas los procesos de integración. Algunas conclusiones adicionales como resultado de la revisión realizada, son:

- El concepto de integración de la cadena de suministro, pretende englobar todos los actores (proveedores, productores, distribuidores y clientes) de la cadena de suministro trabajando en forma conjunta, con objetivos y decisiones comunes, considerando la existencia de un único flujo de productos, y analizando y mejorando los procesos entre cada uno de los actores con el fin de aportar un beneficio global a toda la cadena de suministro.

- La falta de integración en la cadena de suministro genera pronósticos inexactos, baja o excesiva utilización de la capacidad de producción, desconocimiento de la capacidad requerida, exceso o falta de inventarios, efecto látigo, servicio al cliente inadecuado, tiempo de respuesta tardíos al cumplimiento de las ordenes, costos de transporte elevados, costos de fabricación elevados, costos de inventario elevados, errores de envió, errores en la recepción, errores de facturación/ pagos, rendimiento pobre de la cadena.

- El uso de tecnologías de información (E-commerce, E-Business, B2B, B2C, EAI, ERP) permiten mejorar la integración de la cadena de suministro.

- El uso de Outsourcing de servicios (Empresas consultoras), tales como 3PLs, 4PLs, 5PLs y 7PLs, facilitan la integración en la cadena de suministro.

- La integración de la cadena de suministro y las tecnologías de la información es una parte esencial del éxito de cualquier compañía que quiera sobrevivir en el mercado actual. Esta combinación permite tomar decisiones con mayor facilidad y rapidez. 


\section{Referencias Bibliográficas}

[1] Acero E, M. (2012). Administración de la cadena de suministro. Series de gerencia Empresarial.

[2] Ahmed Musa, A. G. (2013). Embedded devices for supply chain aplications: Towards hardware integration of disparate technologies. Expert Systems with Applications.

[3] Arshinder, A. K. (2008). Supply chain coordination: Perspectives, empirical studies and research directions. Int.J. Production Economics, 316-335.

[4] Barbara B. Flynn, B. H. (2010). The impact of supply chain integration on performance. Journal of Operations Management, 58-71.

[5] Benjamin, J. (1989). An Analysis of inventory and transportation cost in a constrained network. Transportation Science. Volumen 23, 177-183.

[6] Bernd Sholz-Reiter, E. M. (2010). Integrating manufacturing and logistic system along global supply chains. Journal of Manufacturing Science and Tecnology, 216-223.

[7] Blumenfield, B. D. (1985). Analizing trade-offs between transportation, inventory and production costs on freight networks. Transportation Research, 361-380.

[8] Blumenfield, B. D. (1991). Synchronizing production and transportation schedules. Transportation Research Part B Volumen 25, 23-37.

[9] Bookbinder, Q. I. (1999). An integrated inventory-transportation system with modified periodic policy for multiple products. European Journal Operation Research, Volumen 115, 245-269.

[10] Brent D. Williams, J. R. (2013). Leveraging supply chain visibility for responsiveness: The moderating role of internal integration. Journal of Operations Management.

[11] Burcu Keskin, H. U. (2010). Integration of strategic and tactical decisions for vendor selection under capacity constraints. Computers \& Operations Research, 2182-2191.

[12] C.F. Cheung, S. K. (2012). A Knowledge-based customization system for supply chain integration. Expert Systems with Applications, 3906-3924.

[13] Cesar, P. (2010). Aplicaciones computacionales en la cadena logistica integrada.

[14] Chee Yew Wonga, S. B. (2011). The contingency effects of environment uncertainty on the relationship between supply chain integration and operational performance. Journal of Operations Management, 604-615.

[15] Chien. (1993). Determining profit-maximizing production/shipping policies in a one-to-one direct shipping, stochastic demand environment. European Journal Operations Research, Volumen 64, 83-102.

[16] Christina W.Y. Wong, C. Y. (2013). The combined effects of internal and external supply chain integration on product innovation. Int. J. Production Economics.

[17] Christos Maravelias, C. S. (2009). Integration of production planning and scheduling. Computers and Chemical Enginnering, 1919-1930.

[18] Claudia Colicchia, G. M. (2013). Building environmental sustainability: empirical evidence from Logistics Service Providers. Journal of Cleaner Production.

[19] Cohen, L. (1988). Strategic analysis of integrated production-distribution systems: Models and methods. Operation Research, Volumen 36, 217-227.

[20] Cornelia Droge, S. V. (2012). Does supply chain integration mediate the relationships between product/process strategy and service performance. Int.J. Production Economics, 250-262. 
[21] Damien. (5 de 15 de 2005). Strategy development processes as determinants of B2b ecommerce performance: A comparative Power. Proquest Education Journals.

[22] Danese, P. (2013). Supplier integration and company performance: A conFigurational view. Omega, 93.

[23] Daniel Prajogo, J. O. (2012). Supply chain integration and performance: The effects of longterm relationships, information tecnology and sharing, and logistics integration. Int.J. Production Economics, 514-522.

[24] Devendra Choudhary, R. S. (2013). Joint decision of procurement lot-size, supplier selection, and carrier selection.

[25] Diana Ballesteros, P. B. (2004). La logística competitiva y la administración de la cadena de suministros. Scientia et Technica Año X No 24.

[26] Edrisi Muñoz, E. C.-G. (2013). Integration of enterprise levels based on an ontological framework. Chemical Engineering Research and Design.

[27] Ellinger, A. (1997). The relationship between integrated logisitcs and customer service.

[28] Erhan Kutanoglu, D. L. (2008). Integrated inventory and transportation mode selection: A service parts logistics system. Transportation Research, 665-683.

[29] Farahani, R. Z. (2013). Competitive supply chain network design: An overview of classification models, solution tecniques and applications.

[30] Ferdinand Jaspers, J. V. (2006). The organizational form of vertical relationship: Dimensions of integration. Industrial Marketing Management, 819-828.

[31] Frank Wiengarten, M. P. (2013). Do a country's logistical capabilities moderate the external integration performance relationship? Journal of Operations Management.

[32] Frolich, W. (2001). Arcs of integration: an international study of supply chain strategies. Journal of Operations Management, 185-200.

[33] Fumero, V. (1997). Integrating distribution, machine assignment and lot sizing via Lagrange Relaxation. International Journal Production Economic, volumen 49, 45-54.

[34] Gang Li, H. Y. (2009). The impact of IT implementation on supply chain integration and performance. Int.J. Production Economics, 125-138.

[35] Giuseppe Confessore, G. G. (2013). A production and logistics network model with multimodal and sustainability considerations.

[36] GunaseKaran. (2004). Information systems in supply chain integration and management. European Journal of Operation Research, 269-265.

[37] Hall. (1996). On the integration of production and Distribution: Economic order and production quantity implications. Transportation Research, Part B volumen 30, 387-403.

[38] lain Dinwoodie, D. M. (2013). Development of a Combined Operational and Strategic Decision Support Model for Offshore Wind. Energy Procedia.

[39] Indranil Bose, R. P. (2008). ERP and SCM system integration. Information and Management, 233-241.

[40] Islam, D. M. (2013). Logistics and supply chain management. Research in Transportation Economics.

[41] J.F., W. (1981). Heuristcs tecniques for simultaneous scheduling of production and distribution in multi-echelon structures. Management Sciences, 336-352.

[42] Jayanth Jayaram, k.-C. T. (2010). Supply chain integration with third-party logistics providers. Int.J. Production Economics, 262-271. 
[43] Jiang Yanhuia, L. X. (2011). A study on supply chain information integration of commodity circulation based on grid. Engineering, 553-557.

[44] Jianming, Y. (2010). Decision optimization analysis on supply chain resource integration in fourth party logistics. Journal of Manufacturing Systems, 121-129.

[45] Kar, A. K. (2013). Revisiting the supplier selection problem: An integrated approach for group decision support.

[46] Katrina Lintukangas, S. (2009). Some issues of supply chain management integration. Journal of Purchasing \& Supply Management, 240-248.

[47] Laureano Jimenez, R. M. (2006). Integration of supply chain management and logistics. Elsevier B.V., 25-35.

[48] Leung, L. C. (2013). Managing third-party logistics under uncertainty: A decision scheme and managerial implications. Int. J. Production Economics.

[49] Marapoulos, K. a. (2005). A theoretical Framework for the Integration of resource aware planning with logistics for the dynamic validation of aggregate plan within a production network. University of Bath, 33-36.

[50] Marcella De Martino, L. E. (2013). Logistics innovation in seaports: An inter-organizational perspective. Research in Transportation Business \& Management.

[51] Marco Perona, N. S. (2004). Integration Techniques in customer-supplier relationship. Int.J. Production Economics, 189-205.

[52] Mc laren, T., Head, M., \& Yufei, Y. (4 de 12 de 2002). Supply chain collaboration alternatives: Understanding the expected costs and benefits. Proquest Education Journal, 348.

[53] Mendoza, A. (2009). The Total Cost of Logistic in Supplier Selection Decisions. Proceeding of the 2009 Industrial Engineering Research Conference, 47-56.

[54] Mendoza, A. (2009). The total cost of logistic in supplier selection decisions.

[55] Min Huang, Y. C. (2013). Fourth party logistics routing problem with fuzzy duration time.

[56] Mithun Sharma, I. M. (2008). 2008. Applied mathematics and computation, 256-265.

[57] Muñoz, L. J. (2006). Integration of supply chain management and logistics: development of an electronic data interchanges for SAP servers. Elsevier B.V., 23-27.

[58] Nagurney, A. (2009). A system-optimization perspective for supply chain network integration: The horizontal merger case. Transportation Research, 1-15.

[59] Neal Juster, A. d. (1997). Enterprise modeling and integration: a taxonomy of seven key aspects. Computer and Industry, 339-359.

[60] Pagell, M. (2004). Understanding the factors that enable and inhibit the integration of operations, purchasing and logistics. Journal of Operations Management, 459-487.

[61] Pamela Danese, P. R. (2013). The impact of supply chain integration on responsiveness: The moderating effect of using an international supplier network. Transportation Research Part $E$.

[62] Pantelis Longinidis, M. G. (2013). Integration of sale and leaseback in the optimal design of supply chain networks.

[63] Paul Cousins, B. M. (2006). The implications of socialization and integration in supply chain management. Journal of Operations Management, 604-620.

[64] Petra Schebert, C. L. (2011). B2B integration in global supply chains: An identification of technical integration scenarios. Journal of Strategic Information Systems, 250-267. 
[65] Piet A. Slats, B. B. (1995). Modeling, Logistic chain. European Journal of Operational Research, $1-20$.

[66] Pineda, C. (2010). Aplicaciones computacionales en la cadena logística integrada. Tesis de grado Especialización Ingeniería de Producción.

[67] Qiu Hong Zhao, S. C. (2010). Integration of inventory and transportation decisions in a logistics system. Transportation Research, 913-925.

[68] Qiu Hong Zhao, S. C. (2010). Integration of inventory and transportation decisions in a logistics system. Transportation Research, 913-925.

[69] Rakesh Nagi, F. P. (2009). Multi-echelon supply chain network design. Proceeding of the 2009 Industrial Engineering Research Conference, 30-35.

[70] Ramanathan, U. (2013). Performance of supply collaboration - A simulation study.

[71] Romano, P. (2001). Co-ordination and integration mechanisms to manage logistics processes across supply networks. Pergamon, 23.

[72] Romano, P. (2003). Co-ordiantion and integration mechanisms to manage logistics processes across supply networks. Journal of Purchasing \& Supply Management, 119-134.

[73] Sabine Fliess, U. B. (2006). Supplier integration - controlling of co-development processes. Industrial marketing Management, 28-44.

[74] Salih Zeki, H. K. (2011). The effect of supply chain integration on information sharing. Procedia Social and Bahavioral Sciences, 1630-1649.

[75] Sanchez, J. E. (2002). Marco Conceptual de la cadena de suministro. México: sanfandila, nro 215.

[76] Sánchez, J. E. (2005). Estado del Arte de los modelos matemáticos para la coordinación de inventarios en la cadena de suministro. México: Sanándola No 281.

[77] Sánchez, J. E. (2006). Coordinación de inventarios en una cadena de suministro del sector automotriz. Mexico: Sanfandila, nro 293.

[78] Sharp, S. G. (1989). A decomposition algorithm for solving the multi-facility production-transportation problem with nonlinear production costs. Econometric, Volumen 38, 450-506.

[79] Speranza, U. (1994). Minimizing transportation and inventory costs for several products on a single link. Operation Research, Volumen 42, 879-893.

[80] Swierczek, A. (2013). The impact of supply chain integration on the "snowball efect" in the transmission of disruptions: An empirical evaluation of the model. Int. J. Production Economics.

[81] Systems, M. a. (2002). Marc Goetschalckx, Carlos Vidal. European Journal of Operational Research, 1-18.

[82] Taco Van Der Vaart, D. P. (2008). A critical review of survey-based research in supply chain integration. Int.J. production Economics, 42-55.

[83] Taskin Gumus, F. G. (2007). Multi-echelon inventory management in supply chain with uncertain demand and lead times: literature review from an operational research perspective. European Journal of Operational Research, 225-239.

[84] Themistocleus, Z. I. (2004). Evaluating the integration of supply chain information systems: A case study. European Journal of Operational Research, 393-405.

[85] Thomas, G. (1996). Coordinated supply chain management. European Journal Operation Research, Volumen $94,1-15$.

[86] Tobias Schoenherra, M. S. (2012). Revisinting the arcs of integration: Cross-validations and extensions. Journal of Operations Management, 99-115. 
[87] Uk Mike. (1996). Integrated Logistics Call in the Revolutionaries.

[88] Vernadat, F. (2002). Enterprise modeling and integration. Annual reviews in control 26, 15-25.

[89] Vijayasarathy, L. R. (2010). Supply integration: An investigation of its multi-dimensionality and relational antecedents. Int.J. Production Economics, 489-950.

[90] Wang Sena, S. P. (2004). Supply chain positioning strategy integration, evaluation, simulation and optimization. Computers \& Industrial Engineering, 781-792.

[91] Wantao Yu, M. A. (2013). The effects of supply chain integration on customer satisfaction and financial performance: An organizational learning perspective. Int. J. Production Economics.

[92] Wei Zhang, D. X. (2013). Integrating the logistics network design with order quantity determination under uncertain customer demands. Expert Systems with Applications.

[93] WookKim, S. (2009). An investigation on the direct and indirect effect of supply chain integration on firm performance. Int.J. Production Economics, 328-346.

[94] Xiande Zhao, B. H. (2008). The impact of power and relationship commitment on the integration between manufacturers and customers in a supply chain. Journal of Operation Management, 368-388.

[95] Xiande Zhaoa, B. H. (2011). The impact of internal integration and relationship commitment on external integration. Journal of Operations Management, 17-32.

[96] Xu, J. (2013). Integrated inventory management and supplier base reduction in a supply chain with multiple uncertainties. European Journal of Operational Research.

[97] Yan Cui, M. H. (2013). Fourth party logistics routing problem model with fuzzy duration time and cost discount. Knowledge-Based Systems.

[98] Yao, J. (2010). Decision optimization analysis on supply chain resource integration in fourth party logistics. Journal of Manufacturing Systems, 121-129.

[99] Yuanqiong. (2012). Supply chain integration service oriented transformation evidence from Chinese equipment manufacturers. Int.J. Production Economics, 791-799.

[100] Yuliang Yaoa, P. E. (2007). Supply chain integration in vendor-managed inventory. Decision Support Systems, 663-667.

\section{Cesar Augusto Pineda Pérez}

Magister en Ingeniería Industrial, Especialista en Ingeniería de Producción e Ingeniero Industrial de la Universidad Distrital Francisco José de Caldas de Bogotá. . Experiencia en diversas empresas del sector industrial. Se ha desempeñado como docente en diversas universidades públicas y privadas en el área de investigación de operaciones, producción y logística.

\section{Cesar Amílcar López Bello}

Magister en Ingeniería Industrial, Universidad de los Andes. Especialista en Ingeniería de Producción, Universidad Distrital. Ingeniero Industrial, Universidad Distrital. Profesor investigador grupo sistemas logísticos de la Universidad de la Sabana. Profesor Asociado, Facultad de Ingeniería Universidad Distrital. Investigador grupo MMAI de la universidad distrital. 